\title{
An interactive image segmentation method for lithological boundary detection: A rapid mapping tool for geologists
}

Yathunanthan Vasuki*1 ${ }^{1}$, Eun-Jung Holden ${ }^{1}$, Peter Kovesi ${ }^{1}$ and Steven Micklethwaite ${ }^{2}$

${ }^{1}$ Centre for Exploration Targeting, School of Earth and Environment, The University of Western Australia, 35 Stirling Highway, Crawley, WA 6009, Australia.

${ }^{2}$ School of Earth, Atmosphere and Environment, Monash University, Clayton, VIC 3800, Australia.

(e-mail: yathunanthan.vasuki@research.uwa.edu.au *; eun-jung.holden@uwa.edu.au; peter.kovesi@uwa.edu.au; steven.micklethwaite@monash.edu).

* Corresponding author. Tel: +61 86488 5807; fax: + 61864881178

\section{HIGHLIGHTS}

- Interactive segmentation based on colour similarity.

- Boundary editing step is used to improve the accuracy of the results for complex images.

- Works as a multi-label image segmentation algorithm.

- Effectively detects the lithological boundaries of geological images.

\section{ABSTRACT}

Large volumes of images are collected by geoscientists using remote sensing platforms. Manual analysis of these images is a time consuming task and there is a need for fast and robust image interpretation tools. In particular the reliable mapping of lithological boundaries is a critical step for geological interpretation. In this contribution we developed an interactive image segmentation algorithm that harnesses the geologist's input and exploits automated image analysis to provide a practical tool for lithology boundary detection, using photographic images of rock surfaces.

In the proposed method, the user is expected to draw rough markings to indicate the locations of different geological units in the image. Image segmentation is performed by segmenting 
regions based on their homogeneity in colour. This results in a high density of segmented regions which are then iteratively merged based on the colour of different geological units and the user input. Finally, a post-processing step allows the user to edit the boundaries.

An experiment was conducted using photographic rock surface images collected by a UAV and a handheld digital camera. The proposed technique was applied to detect lithology boundaries. It was found that the proposed method reduced the interpretation time by a factor of four relative to manual segmentation, while achieving more than $96 \%$ similarity in boundary detection. As a result the proposed method has the potential to provide practical support for interpreting large volume of complex geological images.

\section{KEYWORDS}

Interactive image segmentation

Lithological boundary detection

Region merging

Multi label segmentation

Real time boundary editing

\section{INTRODUCTION}

In recent years image acquisition from aircraft and Unmanned Aerial Vehicles (UAVs) have attracted much attention for remote sensing applications (Harwin and Lucieer, 2012; Turner et al., 2012). UAVs have been enthusiastically adopted by the geoscience community due to their capacity to capture high resolution data remotely and quickly (Bemis et al., 2014; Vollgger and Cruden, 2016). This makes the mapping of exposed rock surfaces for structures, stratigraphy and lithology possible even for locations with limited access. However, UAVs generate large volumes of images, and manual analysis of the captured images is time consuming, warranting the use of automated analysis to provide fast and reproducible results.

Geological mapping using digital photography and remote sensing has been an active area of study, and automated and semi-automated analysis techniques have been applied in various studies (Ferrero et al., 2009, 2011; Kottenstette, 2005; Seers and Hodgetts, 2016; Vasuki et al., 2014). For lithology mapping, there have been a number of studies that used spectral and other 
remotely sensed data to map lithology over large geographical regions. Yu et al. (2012) used machine learning based pattern recognition and image analysis techniques to classify lithological units from multi-spectral satellite images and potential field geophysics data while Abdul-Qadir (2013) used a maximum-likelihood classification method to classify Landsat images. Perez et al (2012) applied a support vector machine to classify lithology using texture features extracted by Gabor filters. Cracknell and Reading (2013) compared the performance of five machine learning approaches (namely random forests, support vector machines, knearest neighbours, naive Bayes and artificial neural networks) and their results showed that the use of random forests is a good choice for lithology classification.

The above mentioned lithology mapping methods focus on characterising and predicting different lithology types using machine learning and classification methods. The study presented in this paper focuses on detecting detailed lithological boundaries from photographic images of rock surfaces using an image analysis approach. Figure 1 shows four photographic rock images used in this study together with their manually mapped lithology. Typical lithological boundaries are contacts between different stratigraphic units, the contact surfaces of an intrusive geological unit or fault lines separating different units. They are associated with discontinuities or changes in visual cues such as colour and texture, due to variations in the mineralogical assemblage in different geological units. Previously, several techniques were used to detect lithological boundaries from remotely sensed data including the rotation variant template matching algorithm (RTM) (Salati et al., 2011; van Ruitenbeek et al., 2008) and the Walsh transform (Maiti and Tiwari, 2005). In the RTM algorithm a user defined a template, which is a row of pixels containing the boundary information, is moved over the grey scale image. The statistical fit is calculated in each position of the image by rotating the template in user defined increments and the angle which has optimal fit is identified. This angle defines the strike of the boundary and the matched pixels are used to define the boundary zone. Salati et al (2011) applied the RTM algorithm to ASTER imagery to detect boundaries between evaporates, marly limestone and sandstone, while Van Ruitenbeek et al (2008) used the RTM algorithm to identify mineral zones in the Pilbara block, Western Australia from hyperspectral imagery. Maiti and Tiwari, (2005) detected lithology boundaries from the German continental deep drilling project borehole well log data using the Walsh transform. Their method identified known lithological units from previous investigations of the study area together with some other finer structures and their presence was confirmed from the geological information. Taye (2011) detected lithology boundaries from aeromagnetic, ASTER, gamma ray and SRTM data 
sets using two methods; an edge detection method (hyperspectral Laplace gradient filtering) and the RTM method. The results show that even though both methods detected the boundaries well, the RTM method performed better than the edge detection method. A pilot study done by Ngcofe and Minnaar (2012) shows that even though the automated technique detects some of the boundaries well, it produced over segmentation in some lithologies. Thus the outcome of their study indicated the use of expert input with automated segmentation was necessary to achieve accurate results. In our approach we have chosen to adopt a similar philosophy of using expert input in conjunction with automated segmentation to identify different rock units in photographic images. 


\section{a}
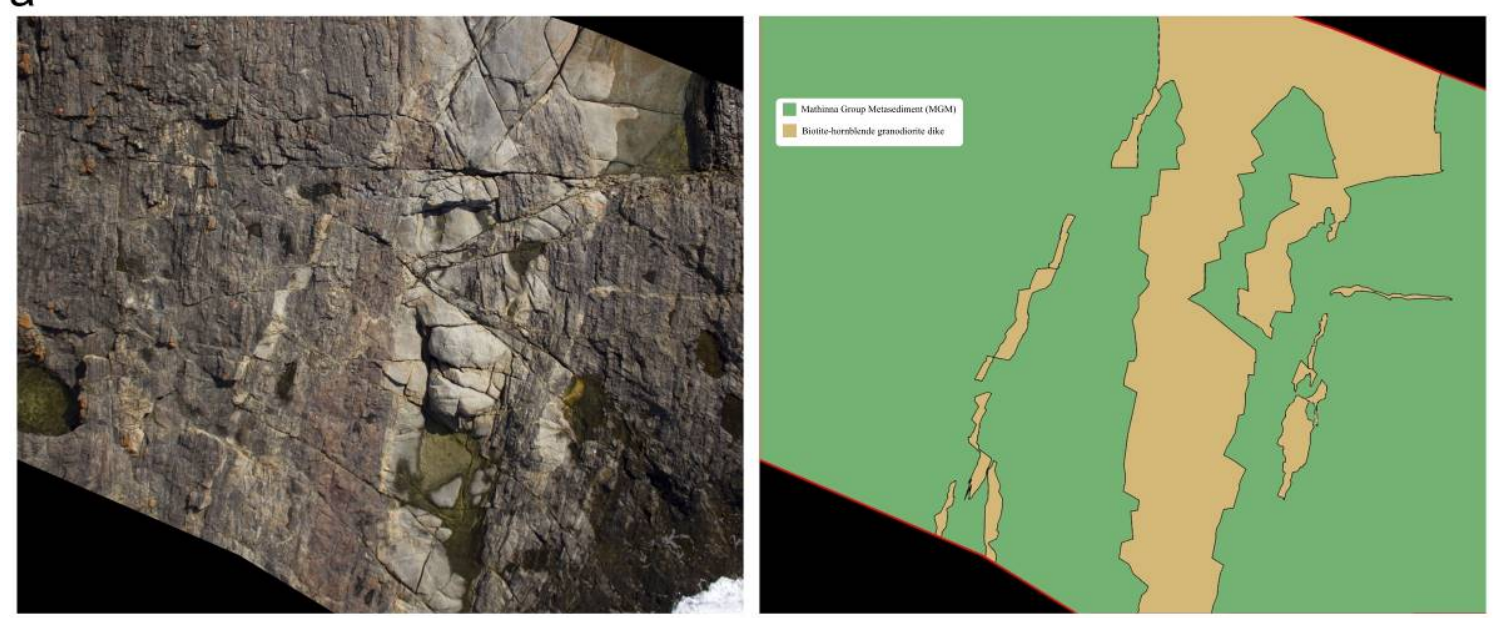

b
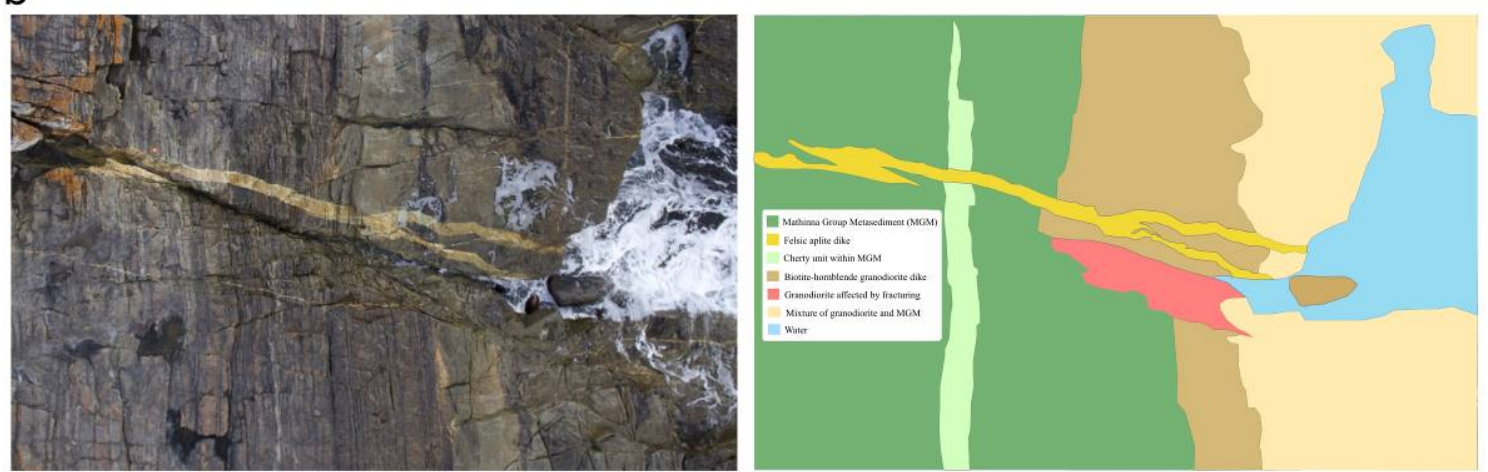

C
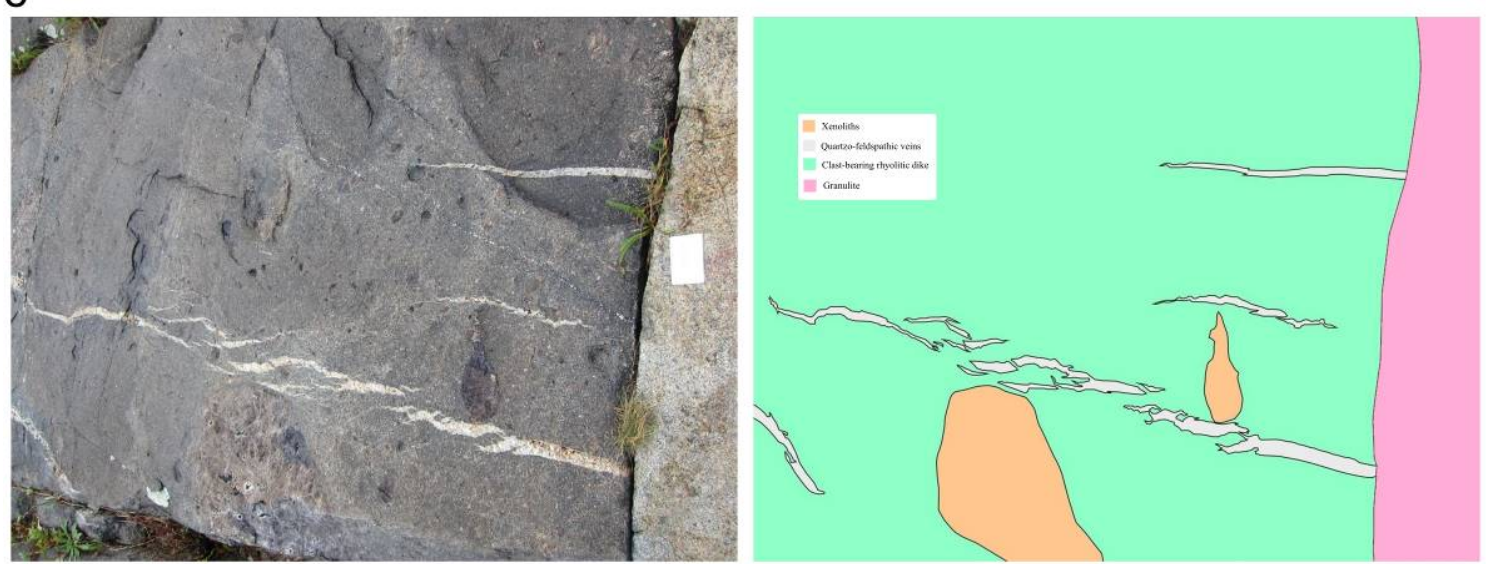

d
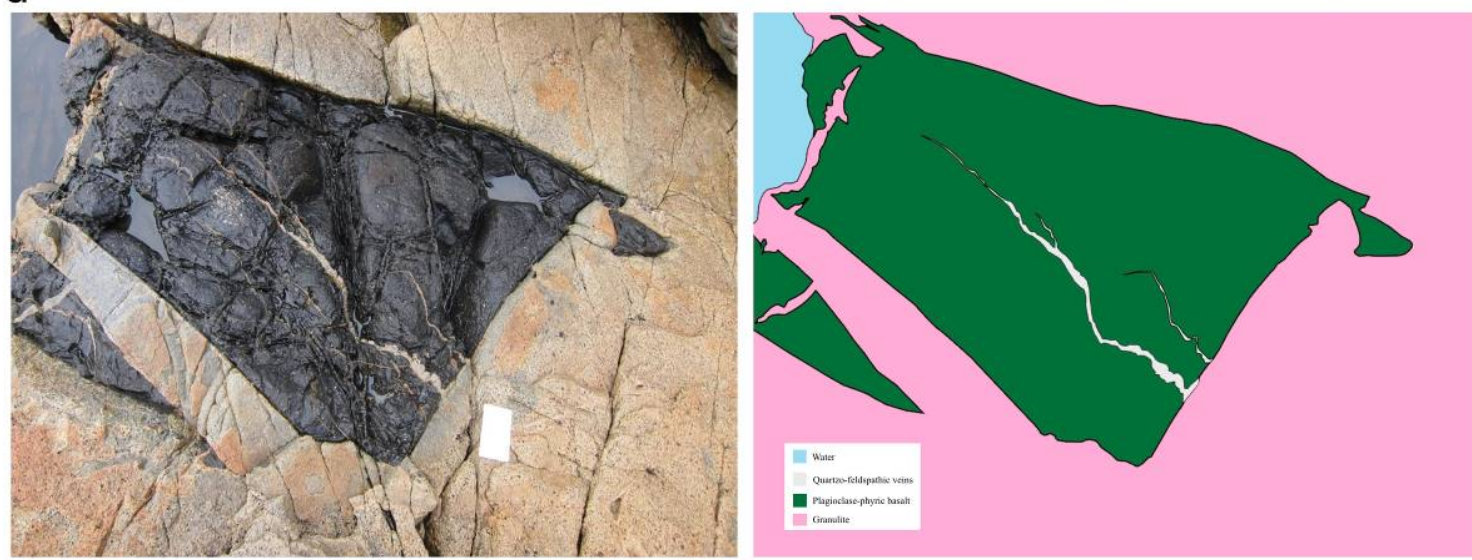
Figure 1. Left Columns: Original high resolution rock images. Right column: Lithological boundaries obtained by manual analysis, the different lithological units are shown in the legend.

\subsection{Image Segmentation}

Automated image segmentation techniques have been used to segment natural images (Martin et al., 2004; Ning et al., 2010), medical images (Grau et al., 2004) and even images from Mars (Gong and Liu, 2012; Song and Shan, 2008). The study by Vantaram and Saber (2012) provides an extensive survey of recent colour image segmentation algorithms. Colour image segmentation algorithms typically use attributes from various colour spaces, for example RGB, LAB, HSV, and LUV (Mignotte, 2008) for segmentation. Other than colour, texture is also an important visual cue for region segmentation (Jones, 1994; Liu and Wang, 2006). A number of studies have used both colour and texture features for image segmentation (Chen et al., 2005; Martin et al., 2004).

There are numerous automated image segmentation techniques available including superpixel (Achanta et al., 2012; Liu et al., 2011), mean-shift (Cheng, 1995; Comaniciu and Meer, 2002) and watershed algorithms (Vincent and Soille, 1991), where neighbouring pixels with homogeneous visual cues are merged to generate segments. However, the outcomes of these algorithms often result in over-segmented regions in complex natural scenes as visual cues in a single object are often not homogeneous. For images of complex natural scenes it is challenging to develop an automated algorithm that can produce an output perceptually equivalent to human analysis. One of the main reasons for this is that an object that needs to be identified as a single segment may not have a homogeneous appearance in terms of colour and/or texture in complex scenes. Thus, some degree of user input is needed to improve the segmentation outcome. Moga and Gabbouj (1998) proposed a marker based watershed algorithm and showed that their interactive method effectively reduces the over segmentation problem found in an automated watershed method. Interactive image segmentation has been proposed and used by many researchers (Boykov and Jolly, 2001; Chen et al., 2011; Dhara and Chanda, 2011; Jung et al., 2014; Li et al., 2004; Ning et al., 2010; Noma et al., 2012; Panagiotakis et al., 2013; Peng et al., 2011; Protiere and Sapiro, 2007; Vezhnevets and Konouchine, 2004; Zhou and Liu, 2012). In these methods, users need to roughly indicate the location of object and background using strokes/curves or bounding boxes. These markers (strokes/curves) give useful information about the user's intention. Thus the rest of the image is effectively segmented to satisfy the user's preference. In their study, Noma et al (2012) 
showed that misclassification in segmentation output may be solved by placing more markers.

Interactive graph cuts (IGC) (Boykov and Jolly, 2001) is a well-known interactive segmentation algorithm, it represents an image using a graph, where the image pixels are represented by nodes of the graph and the edges represent the relationship between adjacent pixels. The min-cut/max-flow algorithm is used to find the globally optimum solution for segmentation. Rother et al (2004) proposed a GrabCut algorithm where the user draws a rectangle around the object region and the colour and edge information are used to segment the image. A graph cut algorithm is then used to find the boundary of the object and this boundary is further refined by using alpha matting. The above mentioned methods use pixel-based operations to interactively segment the image but there are other approaches which apply region-based operations. They use an automated image segmentation algorithm which typically generates over segmented regions, and these regions are then merged based on user input. Ning et al. (2010) developed a maximal similarity region merging method (MSRM), which merges adjacent regions based on local maximum colour similarity. Their algorithm first merges the background regions marked by the user, and then grows the background region by calculating the similarity between it and adjacent regions based on their colour histograms. In a second stage, it merges all the unmarked regions with their adjacent regions if they have maximal similarity over all other adjacent regions. This algorithm has been modified to detect multiple similar colour objects (Chen et al., 2011; Dhara and Chanda, 2011). Jian et al. (2013) extended the MSRM algorithm to segment medical images and they used texture and grey-level similarity to merge the adjacent regions. Graph based approaches have also been proposed for interactively merging initial regions (Noma et al., 2012; Panagiotakis et al., 2013; Peng et al., 2011). Peng et al. (2011) used a localised graph cut algorithm, where in each iteration only the regions adjacent to the user marked regions are processed. Noma et al. (2012) developed a graph based algorithm which merges the initial regions based on colour and spatial information. Long et al (2013) proposed a graph based algorithm, where initial regions are used to build the graph nodes and the min-cut/max-flow algorithm is used to find the merging solution.

The interactive algorithms developed by previous studies provide satisfactory results with most natural images. However none of the methods have been applied to rock or lithology mapping. Most interactive methods do not allow the user to interactively edit the boundaries after producing the results. Rock surface images often have very subtle colour changes near the boundary of different geological units which makes it difficult for even interactive algorithms 
to detect the boundaries correctly. However, accurate tracing of lithology boundaries is important for geological mapping, with significant impact in the interpretations generated for oil and mineral exploration by way of example. Thus, it becomes necessary to allow interactive correction of the analysis results during post-processing stage to produce results which are of practical use to geologist.

This paper presents an Interactive Lithological Boundary Detection (ILBD) method to detect the lithological boundaries of complex geological images. Section 2 of this paper explains the ILBD method in detail and Section 3 presents the outcome of several experiments performed using the ILBD method. In our trial dataset an expert geologist, who visited the study area, manually interpreted the lithological boundaries and the results were used to validate the performance of the proposed algorithm. The time taken for both manual and interactive methods were also calculated for comparison and we compare our method with some other interactive methods.

\section{THE PROPOSED ILBD METHOD}

\subsection{The Proposed Image Segmentation Workflow}

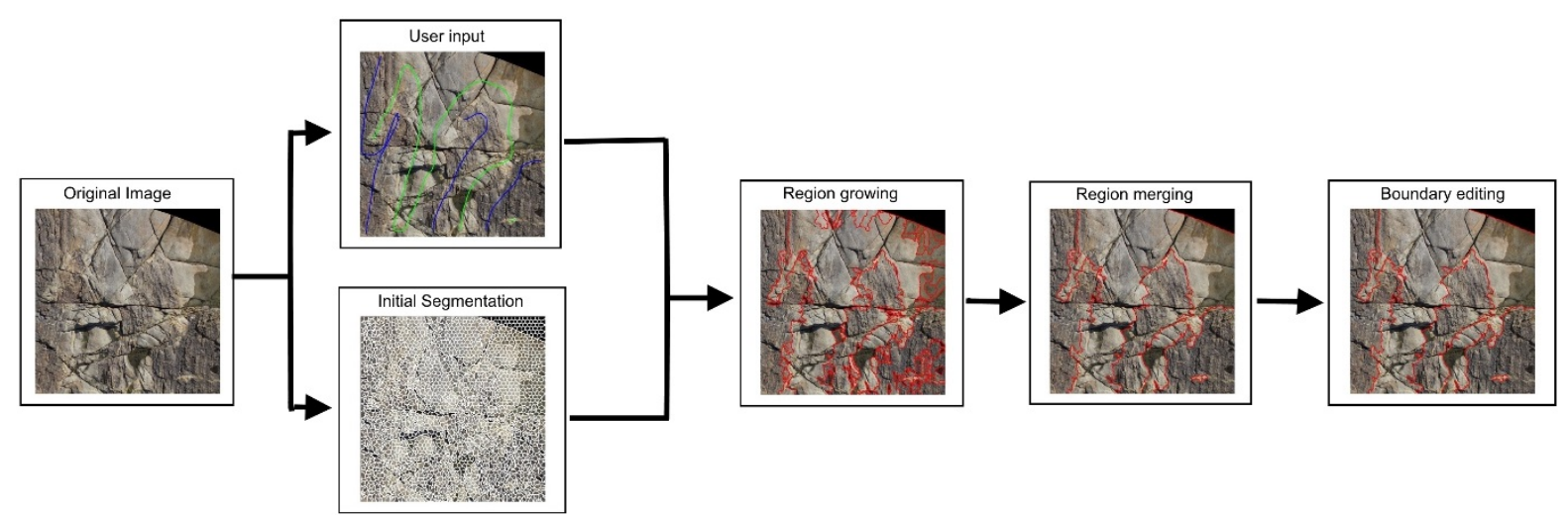

Figure 2. The workflow used to interpret lithological units using the ILBD method, illustrated with a sample area from Figure 1A.

Figure 2 outlines the stages taken using the ILBD method towards finding highly detailed region boundaries which are suitable for lithology mapping. Firstly, the method requires manual input in the form of user drawn markers to guide the multi-label image segmentation between the multiple objects present in a single image. The proposed ILBD method then 
consists of three sequential steps. In the first step, the image is segmented using a superpixel algorithm (Achanta et al., 2012) to form initial regions. Superpixel algorithms group similar image pixels into small patches, this reduces the complexity of subsequent segmentation tasks. These superpixels are then merged with neighbouring superpixels based on their colour similarity. This step optimises the number of regions for further processing while ensuring the preservation of the fine details of region boundaries. The next step is a region growing and merging process that separates the regions specified by the user marked lines. This step uses colour similarity and the user inputs to group the segmented regions from the first step into different lithological units. Finally, a post-processing step (boundary editing step) allows the user to interactively edit the boundaries of the objects defined by the ILBD method.

\subsection{Data}

For this experiment, four digital photographs of rock surface images were used. Two of those photographs (Figure 1a and Figure 1b) are from Piccaninny Point on the east coast of Tasmania, Australia. These photographs were captured using an UAV at an altitude of 30-40m using a Canon 550D DSLR camera resulting in images of approximately $1 \mathrm{~cm}$ resolution (Lucieer et al., 2011). For comparison, two additional photographs were captured from the ground with a Canon S90 handheld digital camera of intrusive exposures from coastal outcrops on the coast of Maine, USA.

\subsection{User Input}

The user is asked to roughly indicate small portions of regions to segment by drawing lines over appropriate areas in the image. These markers help guide the image segmentation process. For maximum segmentation accuracy most of the key features should be covered by these markers (Jung et al., 2014; Ning et al., 2010). The ILBD method is developed as a multi-label segmentation algorithm, thus the user is required to mark connected object/background regions using one continuous line and unconnected objects/background regions need to be marked with separate lines. Figure 3 shows an example rock surface image, where a user mapped the boundaries of biotite-hornblende granodiorite dyke relative to the surrounding metasediment. A green coloured marker indicates the regions associated with granodiorite dyke, and blue markers are used to represent the surrounding metasediment. Note that multiple lines are drawn using same coloured markers to represent separate regions that are not connected. 


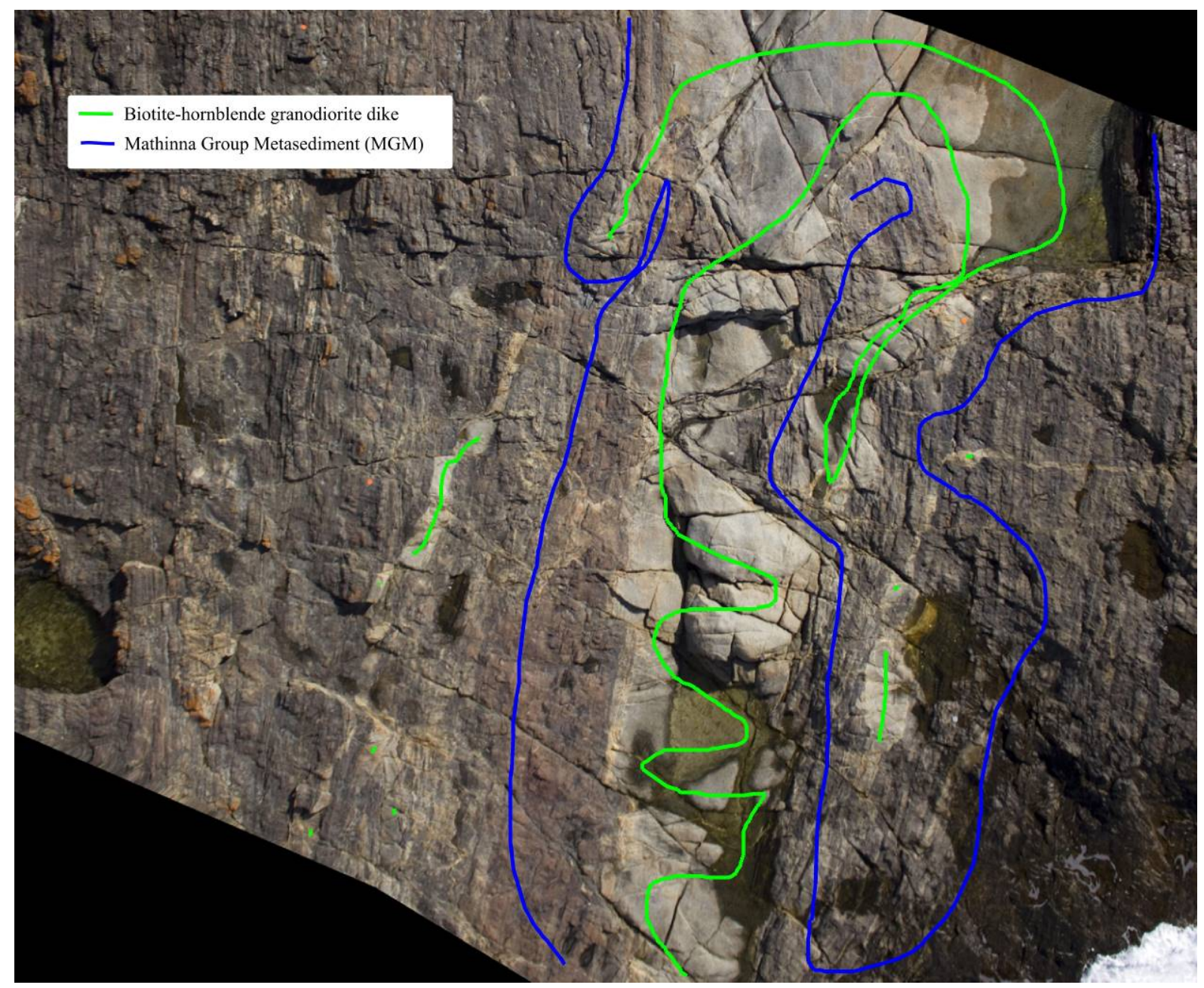

Figure 3. User input. Note that that in this image the different colours of the markers indicate two types of regions to separate- the biotite-hornblende granodiorite dyke marked by green lines and the background metasediments marked by blue.

\subsection{Initial Segmentation}

Pixel-based segmentation: Our image segmentation method first performs a pixel-based segmentation to generate initial segments. The initial segmentation process can employ any of the low-level segmentation methods (Achanta et al., 2012; Cheng, 1995; Liu et al., 2011; Vincent and Soille, 1991), but generating these initial segments such that they preserve the integrity of visual homogeneity within each segment is important as this will affect the detection of detailed boundaries of regions at the later stages of the analysis. Our experiments with complex geological images showed that the Simple Linear Iterative Clustering (SLIC) superpixel algorithm (Achanta et al., 2012) grouped the image pixels effectively to generate initial segments, where their boundaries reflect the lithology boundaries. An example initial 
segmentation output is shown in Figure 5a.

The SLIC superpixel algorithm (Achanta et al., 2012) is based on k-means clustering acting on image pixels in the CIELAB colour space. CIELAB is a perceptual colour space where a colour is represented by its lightness, L, its green-red/magenta opponent value, a and its yellow-blue opponent value b, as shown in figure 4 (Kovesi, 2015). This algorithm is initialised with cluster centres arranged in a regular grid. After that, each pixel in the image is grouped with its nearest cluster centre within a region, where the distance is defined in terms of colour difference in CIELAB space and the spatial distance. The cluster centres are then adjusted to the mean vector of the corresponding cluster. Finally, those pixels not connected to any cluster centres are grouped with their nearest regions (superpixels). In this study a MATLAB implementation of the SLIC superpixel algorithm is used (Kovesi, 2013). For the convenience of algorithm development, we call all the regions which are assigned with a lithological unit as marked region $\mathrm{M}$ and the remaining regions are labelled as non-marked region $\mathrm{N}$. To separate different lithological units, the initial regions, that are connected by the same continuous user drawn line are labelled with a same object name $\left(\mathrm{M}_{1}, \mathrm{M}_{2}, \ldots . ., \mathrm{M}_{\mathrm{m}}\right.$, where $\mathrm{m}$ is the total number of lithological regions which need to be separated).

Optimising the Initial Segmentation Output: The SLIC superpixel algorithm will always produce a fixed (user specified) number of superpixels because it is essentially a k-means process. At this stage the image will be significantly over segmented. To reduce the number of regions we apply a preliminary region merging process, where initial superpixels are merged with their neighbouring superpixels if there is high colour similarity between them. Reducing the number of initial segments minimises the computational cost for the subsequent marker based region growing processes described in Section 2.5.

The region merging process is based on the colour similarity, which is calculated by the colour difference between adjacent superpixels. The colour difference between adjacent superpixels is defined as the Euclidean distance between the median CIELAB colour values of each superpixel. A smaller value of colour distance between two regions represents a higher similarity between those regions. Note that the images and the results are presented in RGB in this paper for visualisation. 


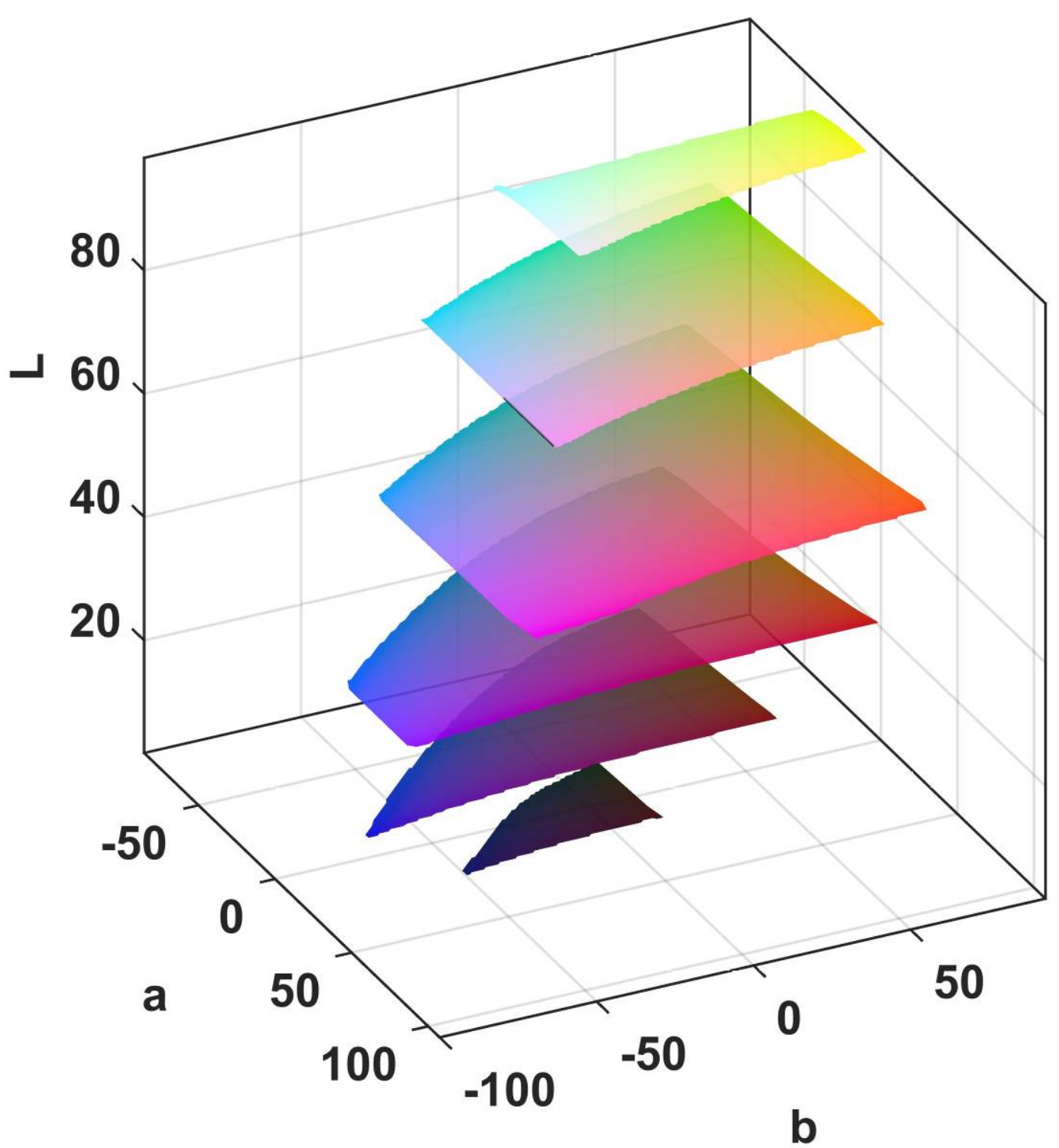

Figure 4. CIELAB colour map.

The colour distance between regions $\mathrm{R}$ and $\mathrm{Q}$ is defined as follows,

$\rho(\mathrm{R}, \mathrm{Q})=\sqrt{\left(R_{L}-Q_{L}\right)^{2}+\left(R_{a}-Q_{a}\right)^{2}+\left(R_{b}-Q_{b}\right)^{2}}$

Where $\mathrm{R}$ and $\mathrm{Q}$ are adjacent regions, $\mathrm{R}_{\mathrm{L}}, \mathrm{R}_{\mathrm{a}}, \mathrm{R}_{\mathrm{b}}$ are the median colour values for the $\mathrm{L}$, $\mathrm{a}$ and b components of region $\mathrm{R}$ and $\mathrm{Q}_{\mathrm{L}}, \mathrm{Q}_{\mathrm{a}}, \mathrm{Q}_{\mathrm{b}}$ are the median colour values for the $\mathrm{L}$, $\mathrm{a}$ and $\mathrm{b}$ components for region Q.

In this step, each initial region is merged with its adjacent region having the minimum colour distance among all its adjacent pairs, provided that those two adjacent regions are not marked 
by the user as two different regions.

Let for a region $Q$, the set of its adjacent regions $S_{Q}=\left\{A_{i}\right\}_{i=1,2 \ldots . . n}$.

$\mathrm{Q}$ and $\mathrm{A}_{\mathrm{j}}$ are defined as minimum colour distance pairs if they satisfy the following condition,

$\rho\left(\mathrm{Q}, \mathrm{A}_{\mathrm{j}}\right)=\min \left(\rho\left(\mathrm{Q}, \mathrm{A}_{\mathrm{i}}\right)\right){ }_{\mathrm{i}=1,2 . ., . ., \mathrm{n}}$

Where $A_{j}$ is one of the adjacent regions of $Q$.

This process reduces the number of regions in the subsequent step. While this process can be applied in multiple iterations to further reduce the number of regions, the resulting regions are to be used as input to the subsequent region growing process, which integrates regions based on markers provided by the user. Thus, it is important to balance reducing the number of regions with preserving the integrity of homogeneity in each region which can affect fine details of region boundaries. We empirically determined that two iterations for this merging step produces a good balance that satisfies these requirements (Figure 5b). This optimisation step is particularly useful for complex images such as geological images, where the initial segmentation algorithm groups the image into a large number of small regions as shown in Figure 5a. In this example, 10341 initial regions are reduced to 2135 regions after the optimisation step. The segmented regions from the optimisation step are labelled individually and are used in the subsequent region growing process.
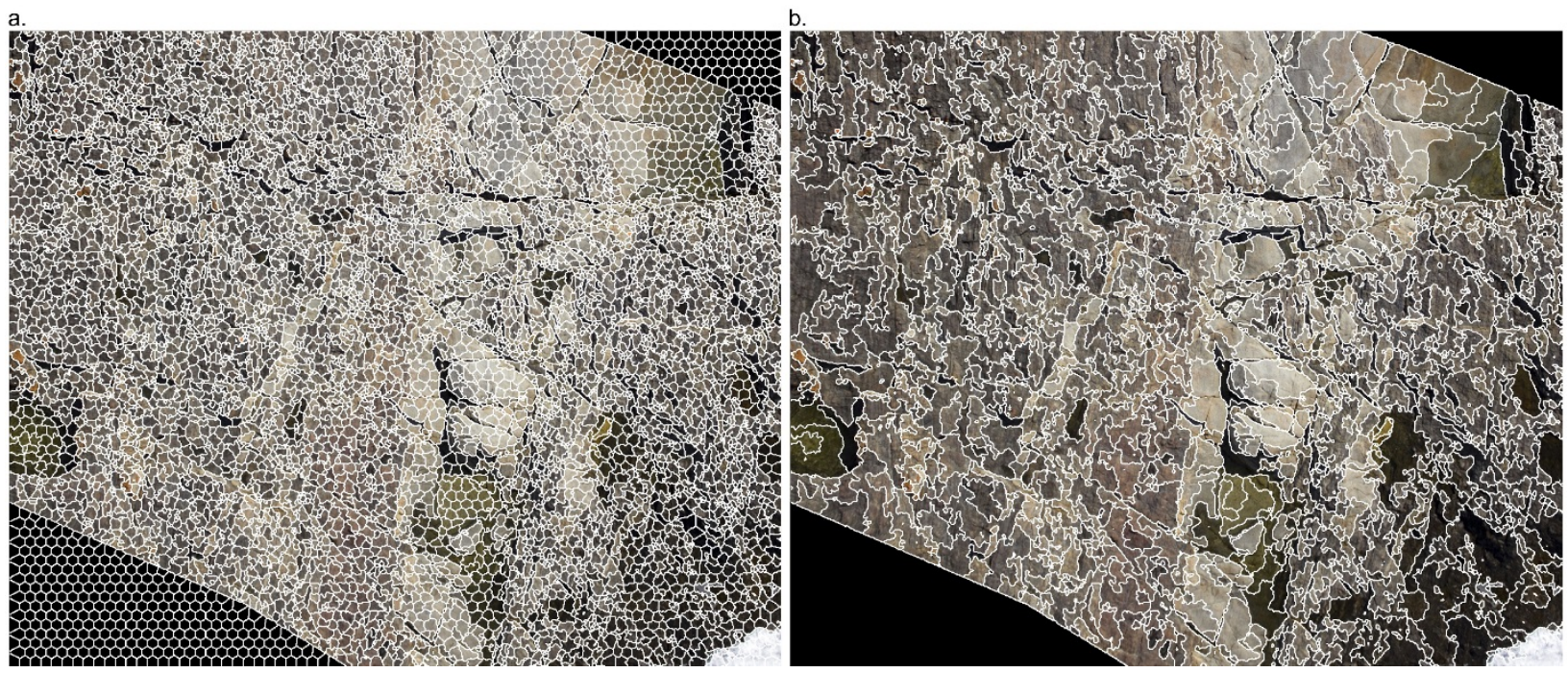

Figure 5. (a) Initial regions (super pixels) generated by the SLIC superpixel algorithm applied to the image previously shown in Figure 1. (b) Regions generated as a result of the optimization 
step, which merged some of the initial regions in (a).

\subsection{Marker-based Object Segmentation for Lithology Boundary Detection}

Our initial segmentation produces image regions where pixels in each regions have similar colour characteristics. The aim of the marker based region growing process is to group those regions into lithological units which have been marked by the user with a specific aim to find their boundaries. To detect the boundaries of different lithological units, all of the segmented regions should be assigned with the correct label of the lithological unit.

Previously two-step strategies have been effectively used for image segmentation. Ning et al., (2010) proposed a method which merges all the initial regions belonging to the background to isolate the object from the image. In the first step, to grow the background region, their method merges each user marked background region with one of its non-marked adjacent regions that has maximum colour similarity. Then in the second step all the remaining regions are merged with their adjacent regions based on colour similarity. This process is iteratively performed to extract the object from the image. We also use a two-step algorithm for image segmentation. In the first step, region growing is iteratively applied to the initially marked 'seed' regions. In the second step, a region merging algorithm, similar to the optimising initial segmentation output step (described in 2.4) is used to merge the regions iteratively until all the lithological boundaries are detected.

\subsubsection{Region Growing from Seed regions}

For each region $P(P \in M)$, its set of adjacent regions $S_{P}=\left\{B_{i}\right\}_{i=1,2,, \ldots k}$ are identified. Then,

for each $\mathrm{B}_{\mathrm{i}}$, if it is not a marked region (i.e $\mathrm{B}_{\mathrm{i}} \in \mathrm{N}$ ), its set of adjacent regions $S_{B_{i}}=\left\{S_{j}^{B_{i}}\right\}_{\mathrm{j}}=$ 1,2,,... are formed. It is obvious that $\mathrm{P} \in S_{B_{i}}$. The colour distance between region $\mathrm{B}_{\mathrm{i}}$ and all its adjacent regions $S_{B_{i}}$, that is $\rho\left(\mathrm{B}_{\mathrm{i}}, S_{j}^{B_{i}}\right)$ are calculated using Equation (1) described in section 2.4. If $P$ and $B_{i}$ satisfy Equation (2), then there is a high probability that the non-marked region $\mathrm{B}_{\mathrm{i}}$ belongs to the same object region $\mathrm{P}$. Thus, $\mathrm{B}_{\mathrm{i}}$ is merged with $\mathrm{P}$ and assigned to the same label as region $\mathrm{P}$. If region $\mathrm{B}_{\mathrm{i}}$ has a minimum colour distance with any region other than $\mathrm{P}$, then $\mathrm{B}_{\mathrm{i}}$ and $\mathrm{P}$ will not be merged. This step is performed iteratively. After each iteration, the labels of the regions are adjusted accordingly and the set of $\mathrm{M}$ (marked regions) and $\mathrm{N}$ (non-marked 
regions) updated. This process stops when the set of marked regions $M$ stops changing. The region growing step of our ILBD method is summarised as below,

Algorithm 1. Region growing step

Input: Labelled regions from pre-processing step

1. For each region $P \in M$, compute its adjacent regions $S_{P}=\left\{B_{i}\right\}_{i=1,2, \ldots k}$

2. For each $\mathrm{B}_{\mathrm{i}} \in \mathrm{N}$, compute its adjacent regions $S_{B_{i}}=\left\{S_{j}^{B_{i}}\right\} \mathrm{j}=1,2,3, \ldots$. Noting that $\mathrm{P}$ $\in S_{B_{i}}$

3. Calculate the colour distance between region $\mathrm{B}_{\mathrm{i}}$ and $S_{B_{i}}$ using equation (1) and if $\mathrm{P}$ and $\mathrm{B}_{\mathrm{i}}$ satisfy equation (2), then merge $\mathrm{B}_{\mathrm{i}}$ with $\mathrm{P}$

4. Update the labels of merged regions and $\mathrm{N}$ accordingly

5. If the regions in $M$ no longer changes end this process; otherwise go back to (1-1).

After this region growing step, some of the non-marked regions will be merged with marked regions. However, there may be some remaining non-marked regions that are surrounded by a marked region as shown in Figure 6a. These regions are merged with their surrounding marked region and the labels are updated accordingly. Nevertheless, after this region growing step, there may still be non-marked regions remaining as shown in Figure 6b. These regions are not merged by the region growing algorithm since they have higher colour similarity with other non-marked regions than their similarity with the marked regions.
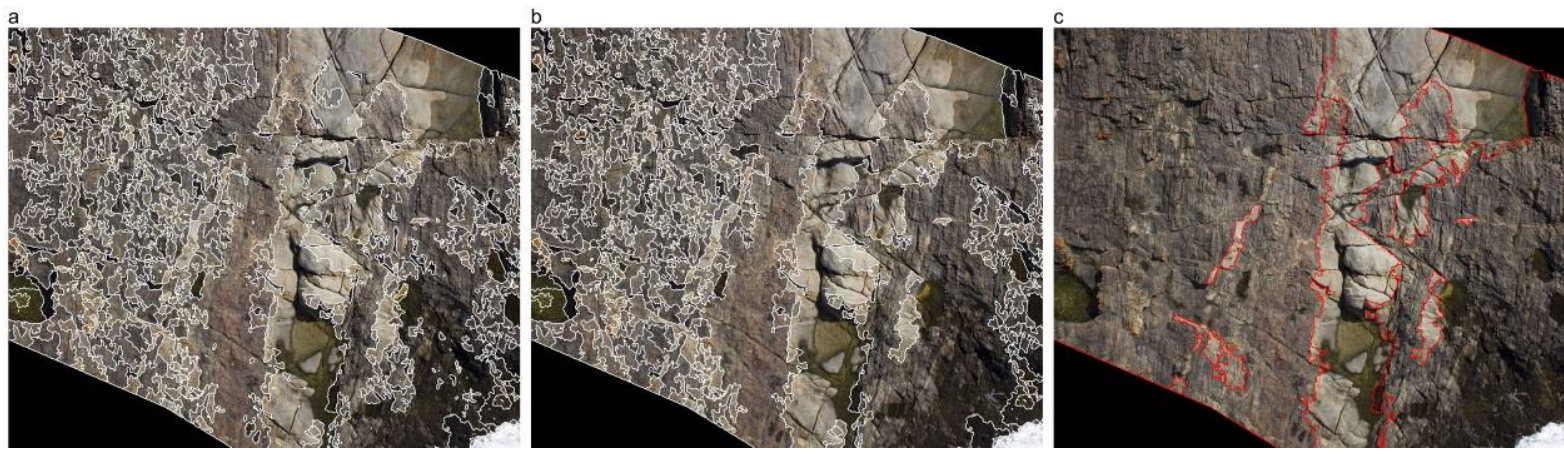

Figure 6. (a) Results after region growing before filling the enclosed regions (b) Results after region growing (c) Final segmentation

\subsubsection{Region merging algorithm}


All the remaining non-marked regions can be merged with their adjacent regions that have the maximum similarity (minimum colour distance) out of all the adjacent pairs as proposed in MSRM (Ning et al., 2010). However, this process forces each region to merge with at least one of its adjacent regions and this may result in merging of regions with low similarity. In order to avoid this, a threshold $\lambda$ is introduced in our method. Nonetheless, to preserve the lithological boundaries, all the non-marked regions should be merged with one of the marked regions. Thus, this step is repeated iteratively until all the non-marked regions are merged with a marked region.

The threshold $\lambda$ is defined as $\lambda=\mu$.T, where $\mu$ is a user defined threshold which is the percentage of adjacent regions which need to be merged in each iteration and $\mathrm{T}$ is the total number of adjacent pairs. The value of $\lambda$ will vary in each iteration as regions are being merged and the total number of adjacent regions changes. A typical value for $\mu$ that we have found useful is around $20 \%$.

For a region $R$, let the set of its adjacent regions be $S_{R}=\left\{A_{i}\right\}_{i=1,2, \ldots n}$. The region $R$ is merged with $A_{j} \in S_{R}$, if all of the following conditions are satisfied: (a) the colour distance between $R$ and $\mathrm{A}_{\mathrm{j}}$, defined as $\rho\left(\mathrm{R}, \mathrm{A}_{\mathrm{j}}\right)$, is the lowest among the colour distances between $\mathrm{R}$ and all of its adjacent pairs in $\mathrm{S}_{\mathrm{R}}$ (b) their colour distance score is among the top $\lambda$ values; and (c) $\mathrm{R}$ and $\mathrm{A}_{\mathrm{j}}$ do not belong to two different user marked regions (i.e if $R \in M_{p}$ then $A_{j} \notin M_{k}(k \neq p)$, where $\mathrm{M}_{\mathrm{p}}$ and $\mathrm{M}_{\mathrm{k}}$ are marked by the user as two different objects). This algorithm is shown below,

\section{Algorithm 2. Merging step}

Input: output from region growing step, threshold $\mu$ which is a user defined percentage

1. For each region $R$ in the image, identify its adjacent regions $S_{R}=\left\{A_{i}\right\}_{i=1,2,3, \ldots n}$

2. For each region $\mathrm{R}$ in the image, calculate the colour distance between region $\mathrm{R}$ and $A_{i}$ using equation (1) and find the pair with minimum colour distance using equation (2)

3. Sort the colour distance scores in ascending order

4. Calculate $\lambda=\mu \mathrm{T}$ ( $\mathrm{T}$ is the total number of adjacent region pairs)

5. Get the top $\lambda$ colour distance scores from the sorted scores. Merge these adjacent pairs if they have not been marked as different regions by the user. 
6. Update the labels of merged regions and $\mathrm{N}$.

7. If $\mathrm{N}$ is empty then end this step; otherwise go back to (2-1).

After this region merging stage the image will be separated into a number of regions, equal to the number of regions defined by the initial markings made by the user. Different values for $\mu$ were tested for various images and the analysis showed $\mu=0.2$ (20\%) gave the best performance for most of the images used in this paper. The final results obtained after these steps are shown in Figure 6c.

\subsection{Boundary editing}

The output of the image segmentation algorithm may vary depending on the level of coverage of user marked lines that were used to represent the distribution of different lithological units, as well as the level of colour homogeneity within the units. Thus, for our system to be used as a practical tool for geologists, it is important that the boundaries can be easily edited in a post processing step. A Graphical User Interface has been designed to allow interactive post-editing of the region boundaries. To edit the object boundary, the user needs to roughly sketch the correction. This is done by selecting the region that needs to be changed by pressing the left mouse button and dragging the mouse pointer through the areas that need to be added to the selected region. Then, the boundary editing algorithm modifies the segmentation based on the user drawn sketches using the original superpixel image segmentation output which represents the most primitive regions used in our segmentation algorithm. The user can view the edited results and continue this process until they achieve a satisfactory result (Figure 7).
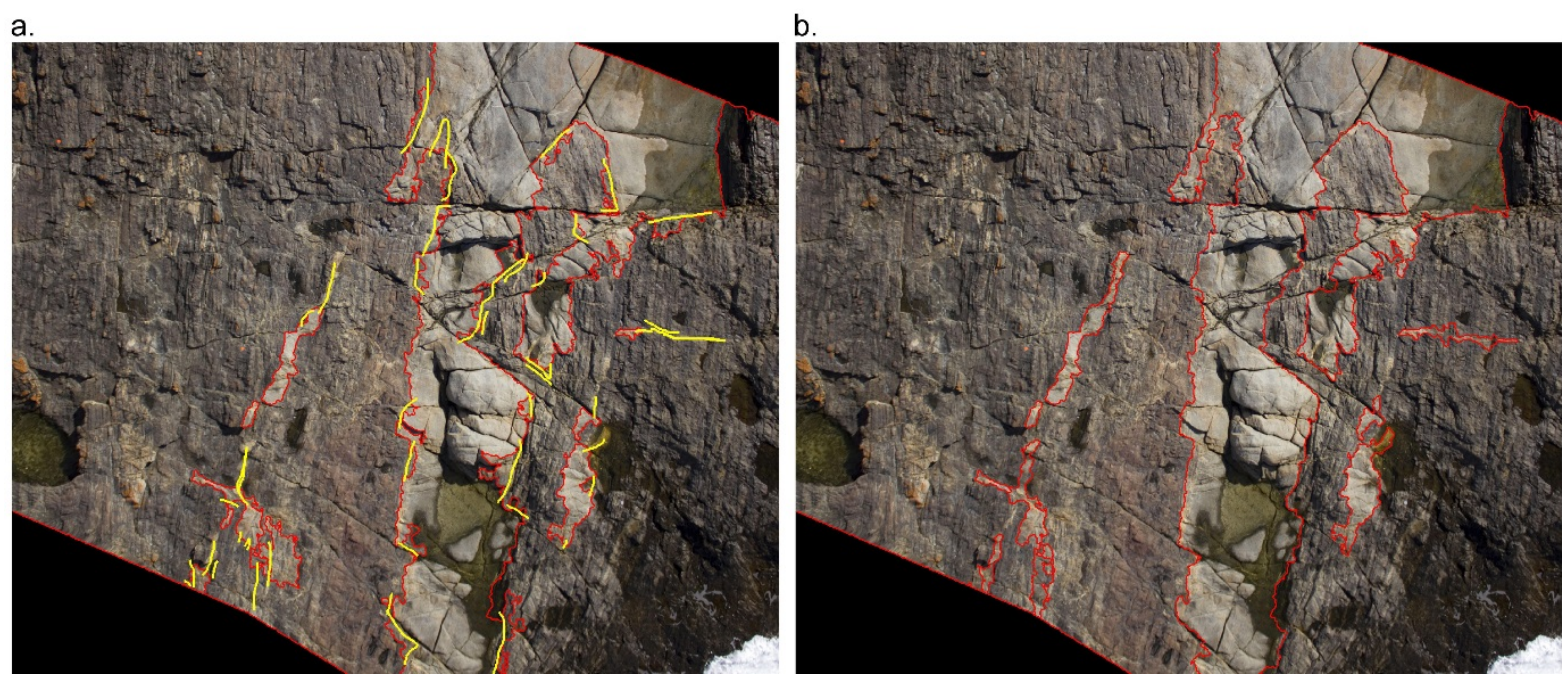
Figure 7. (a) User input (yellow) used to edit the boundary. (b) Lithological boundaries obtained after boundary editing.

Implementation: The proposed method was implemented in a MATLAB environment, and a desktop with Windows 764 bit, Intel core i7, 3.4 GHz CPU and 32 GB RAM was used to run this algorithm.

\section{EXPERIMENTAL RESULTS}

Experiments were conducted to demonstrate the effectiveness of our ILBD method for lithology mapping of two UAV collected images shown in Figure 1. The aim was to map different lithology units present in those images. These images were also manually mapped by an expert geologist, and used to evaluate the results of our method. In addition, we compared the performance of the proposed method with other well-known methods, namely MSRM (Ning et al., 2010), DG (Noma et al., 2012) and IGC (Boykov and Jolly, 2001) using natural scene images to test the applicability of the ILBD method to non-geological images.

\subsection{Manual Interpretation}

ArcMap 10.2.1 and ArcCatalog were used to generate a digital map by non-automatic methods (see Figure 1). A standard geological digitising protocol was followed, whereby three separate digital vector files (SHAPE_FILES) were created in ArcCatalog to delineate the boundary of the map area, the different lithological contacts and the geological units present in the outcrop. These were then opened in ArcMap for digitisation. The map boundary and the different lithological contacts were traced using the Editing Tool, generating a series of line vectors. Polygons were subsequently constructed from the traces of the lithological boundaries and defined as appropriate geological units.

\subsection{Quantitative Performance Measure}

For quantitative analysis of the performance of ILBD method, the accuracy of the boundary is calculated (Jung et al., 2014). Accuracy is a measure of correctly classified pixels.

\subsection{Lithology mapping using proposed method}



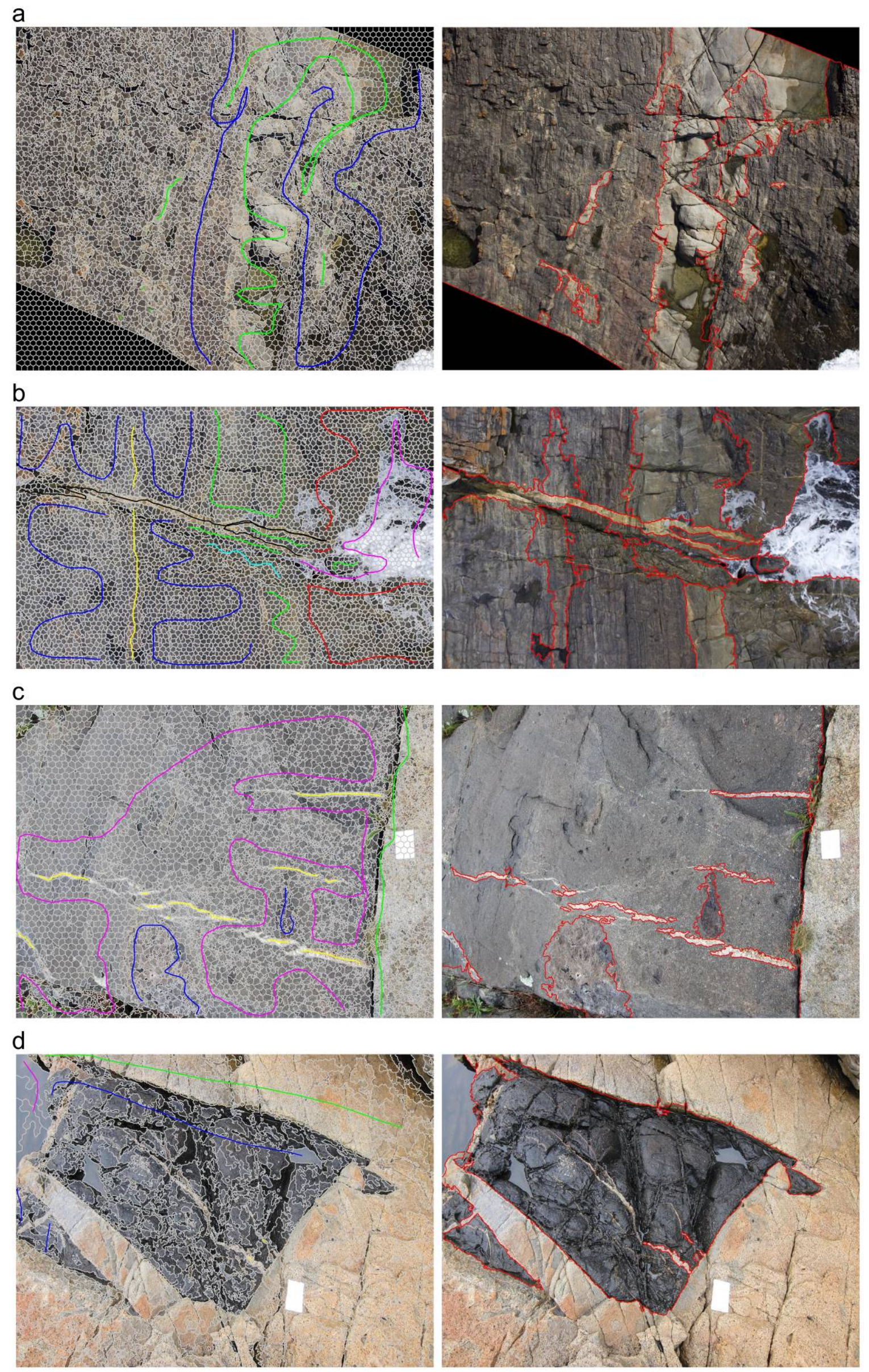
Figure 8. Left column: Initial super pixels and user markings, different colours are used to mark different lithological units. Right column: Lithological boundaries detected by the ILBD method without the boundary editing step. In Figure 8(a) and (b) the biotite-hornblende granodiorite dyke is marked by green lines, mathinna group metasediments marked by blue, felsic aplite dike marked by black, cherty units marked by yellow, granodiorite marked by cyan, the water area marked by magenta, and the mixture of granodiorite and metasediments marked by red lines. In Figure 8(c) xenoliths are marked by blue, quartzo-feldspathic veins marked by yellow, clast-bearing rhyolitic dike marked by magenta, and granulite marked by green lines. In Figure 8(d) quartzo-feldspathic veins are marked by yellow, plagioclase-phyric basalt marked by blue, granulite marked by green lines, and the water is marked by magenta lines.

Figure 8 shows the initial segments of the four geological images used in this study, together with the user markings. The time taken by the proposed algorithm to produce the results shown in Figure 8 (a), (b), (c) and (d) are $263 \mathrm{sec}, 78 \mathrm{sec}, 287 \mathrm{sec}$ and $263 \mathrm{sec}$ respectively. The total time taken to produce the results shown in Figure 8 was recorded. This included the time taken to place user markers and process the image using the ILBD method. The same user edited the boundaries using the interactive boundary editing method and the total time was determined, including the time taken to place boundary markers. Please note that different users may need different amount of time to place the initial markers and boundary markers. Figure 9 shows the final boundary edited result. For visual comparison of the results, the outline of the object boundaries detected by the ILBD method is overlaid on top of the manually interpreted image. The total time taken by manual and interactive mapping is shown in Table 1 and the accuracy of results are reported as a percentage of correctly classified pixels. The results presented in Table 1 demonstrate that with the boundary editing step the ILBD method reduced the time taken for interpretation by more than the factor of four when compared to manual interpretation while achieving more than $90 \%$ accuracy. The accuracy increased to more than $96 \%$ when the boundary editing step was used. 
a

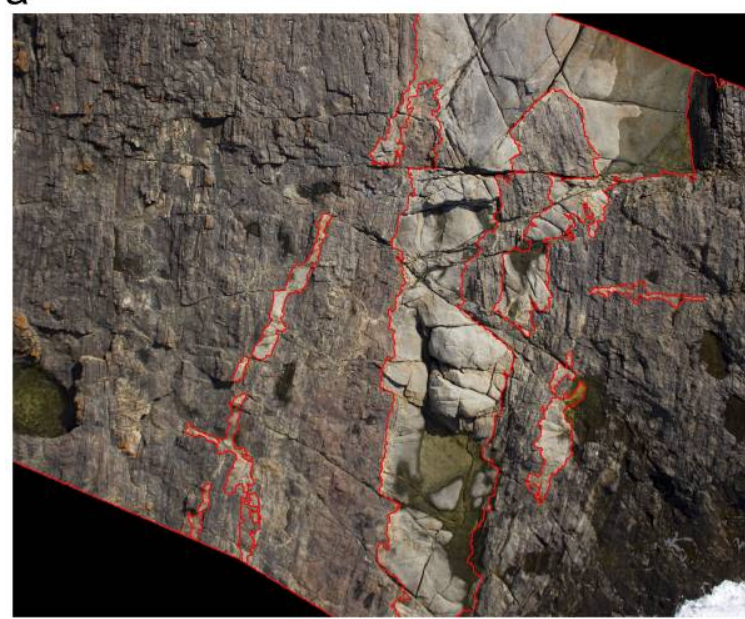

b

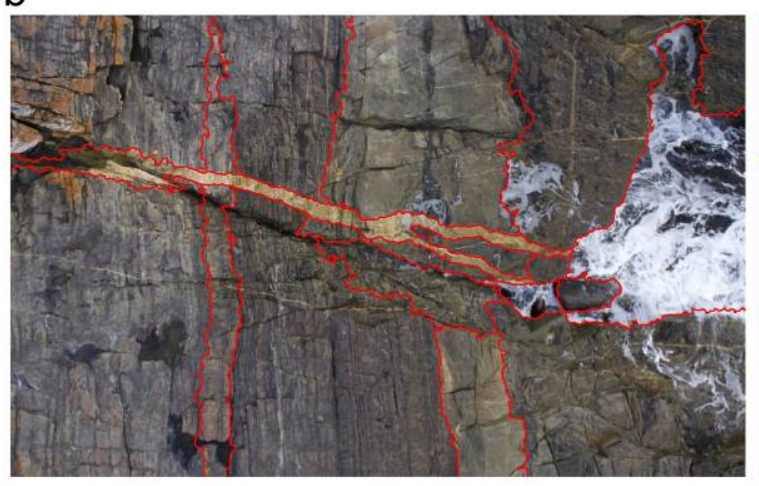

C

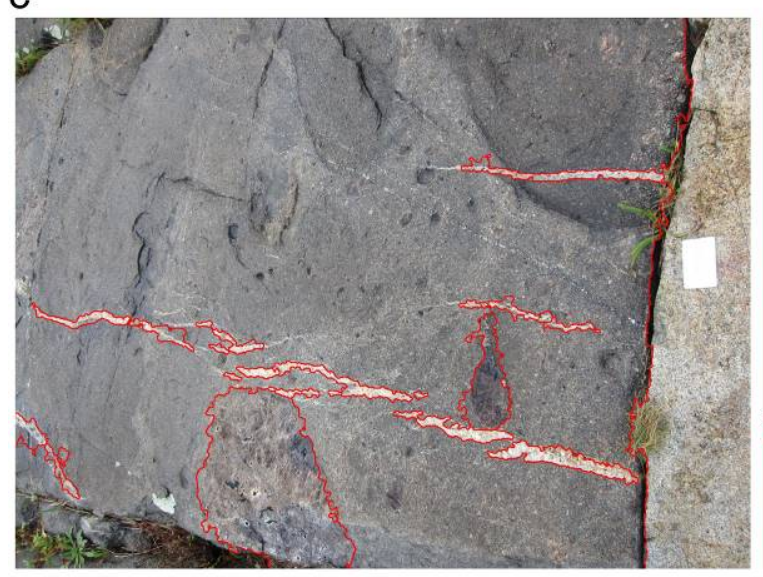

d

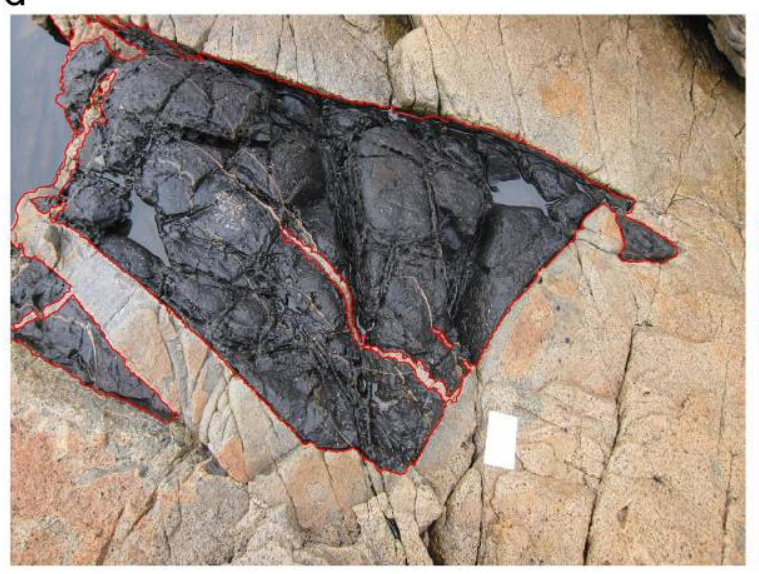

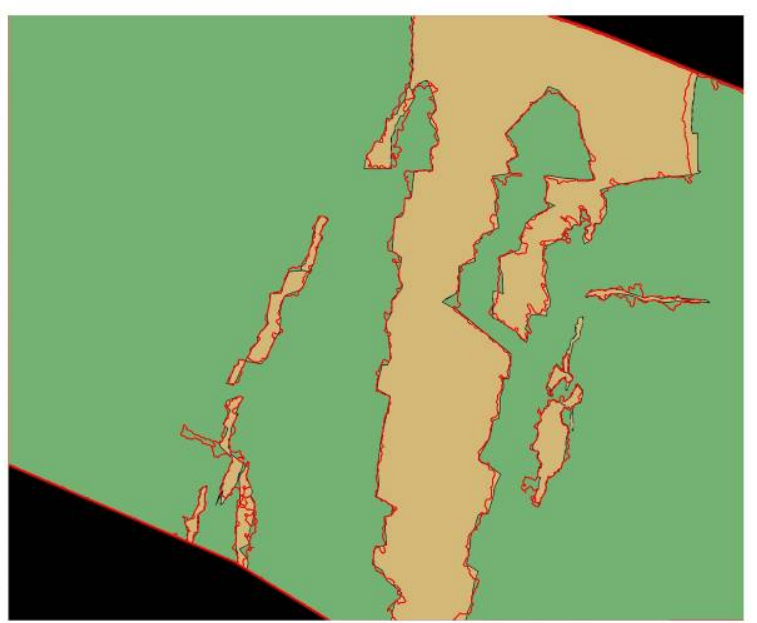
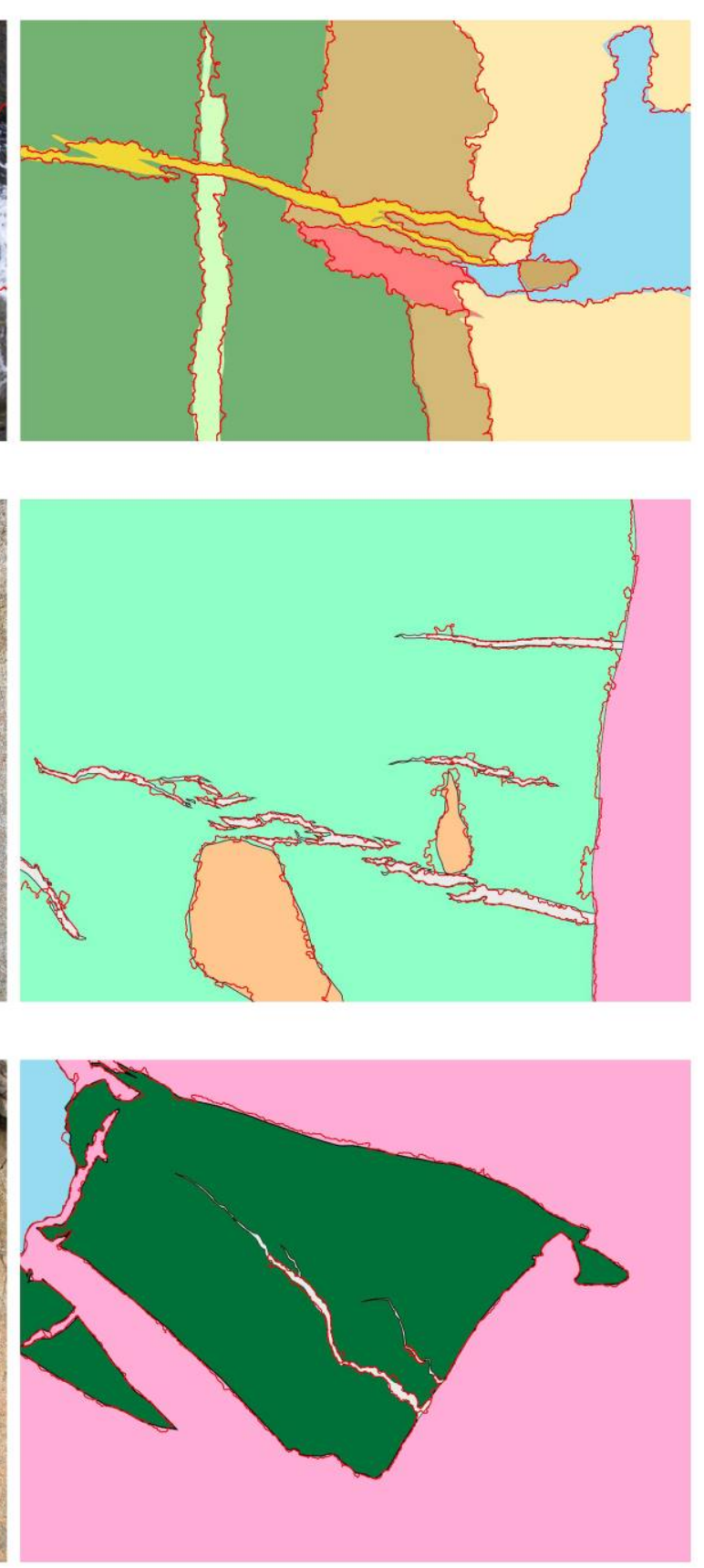
Figure 9. Left column: Lithological boundaries obtained after boundary editing. Right column: Lithological boundaries obtained by the ILBD method overlaid on top of the manual interpretation, where different colours indicate different types of rock. For explanation of the different rock types please refer to the legend in Figure 1.

Table 1. Performance of the proposed algorithm for lithological boundary detection in the two example images.

\begin{tabular}{cccccc}
\hline & \multicolumn{2}{l}{ ILBD without boundary editing } & \multicolumn{2}{l}{ ILBD with boundary editing } & Manual \\
\hline & Accuracy & Time & Accuracy & Time & Time \\
\hline Figure 9a & $95.5 \%$ & $8 \mathrm{~min}$ & $97.9 \%$ & $11 \mathrm{~min}$ & $95 \mathrm{~min}$ \\
Figure 9b & $90.8 \%$ & $5 \mathrm{~min}$ & $96.5 \%$ & $11 \mathrm{~min}$ & $70 \mathrm{~min}$ \\
Figure 9c & $95.1 \%$ & $10 \mathrm{~min}$ & $96.2 \%$ & $14 \mathrm{~min}$ & $90 \mathrm{~min}$ \\
Figure 9d & $98 \%$ & $6 \mathrm{~min}$ & $98.6 \%$ & $8 \mathrm{~min}$ & $35 \mathrm{~min}$ \\
\hline
\end{tabular}

\subsection{Comparison with other methods}

The proposed segmentation algorithm is specifically developed to map the lithological boundaries from geological images. However, the proposed method outperforms state-of-theart methods when segmenting non-geological images as well. Experiments were performed and the results are compared with interactive image segmentation algorithms namely MSRM (Ning et al., 2010), DG (Noma et al., 2012) and IGC (Boykov and Jolly, 2001). For the MSRM and DG methods the source codes published by their respective authors were used and for IGC we used the implementation of McGuinness and O’Connor, (2008). For each image tested, the same input markers were used for all of the algorithms. For the DG method we used its default initial segmentation method, the watershed transform proposed by Vincent and Soille, (1991) and its post-processing step. MSRM and IGC are single object extraction algorithms thus we did not use it with the rock surface images in section 3.4.1.

\subsubsection{Experiments with rock surface images}

In this section we compare the ILBD algorithm with another multi label segmentation algorithm DG (Noma et al., 2012). The DG algorithm was used to extract different lithological units from the rock surface images used in this paper with the input markers shown in Figure 8. The boundaries detected by the DG algorithm are shown in Figure 10 and it achieved 
accuracy of 78.5\%, 91\%, 94.1\% and 67.8\% for Figure 10 (a),(b),(c) and (d) respectively. The proposed ILBD algorithm achieved higher accuracy for all the images with the boundary editing step. DG performed slightly better than the proposed algorithm for Figure 1b when the boundary editing step of ILBD is not used. However once the boundary editing step was applied the ILBD algorithm achieved 96.5\% accuracy for that image.

a

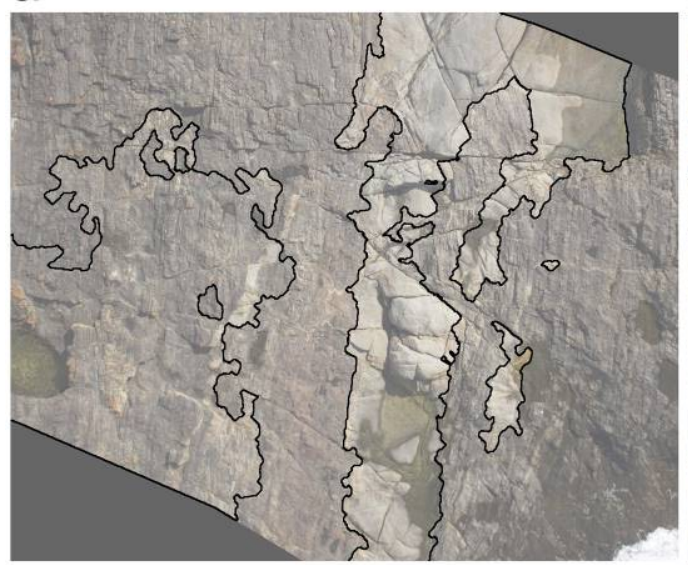

C

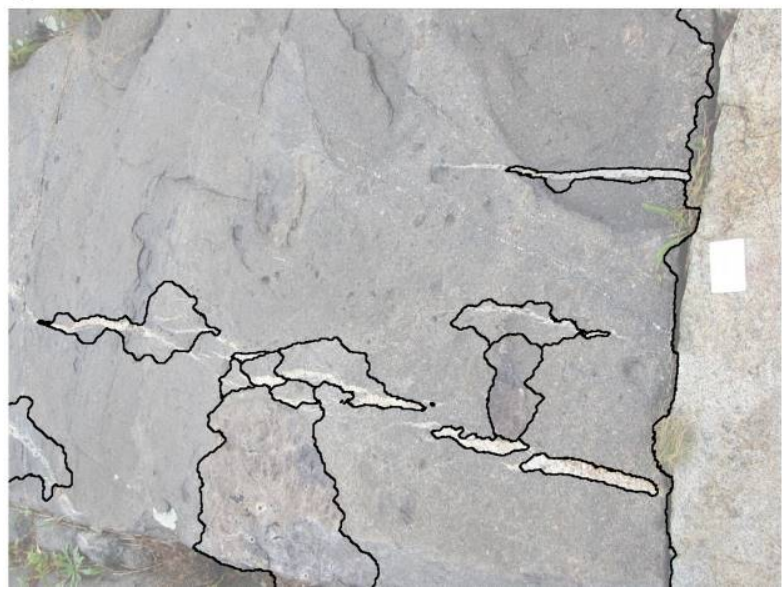

b

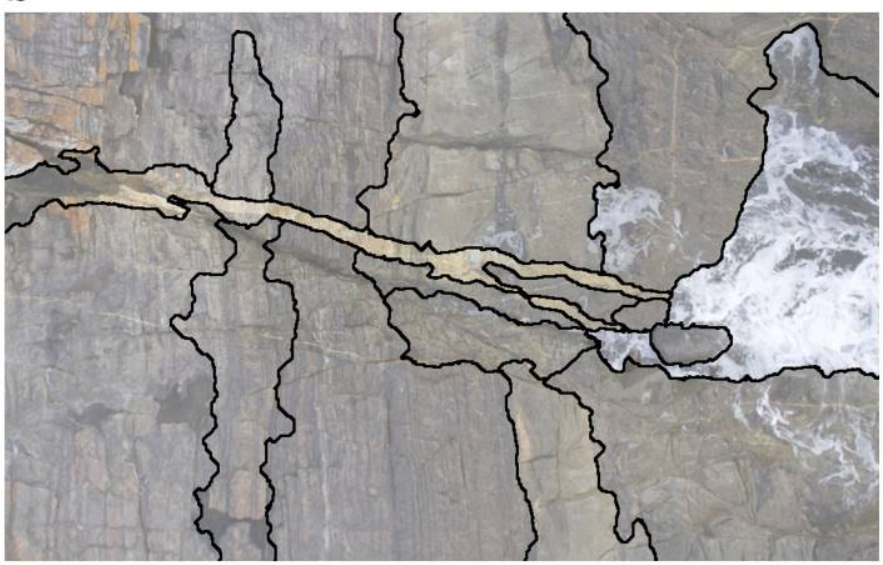

d

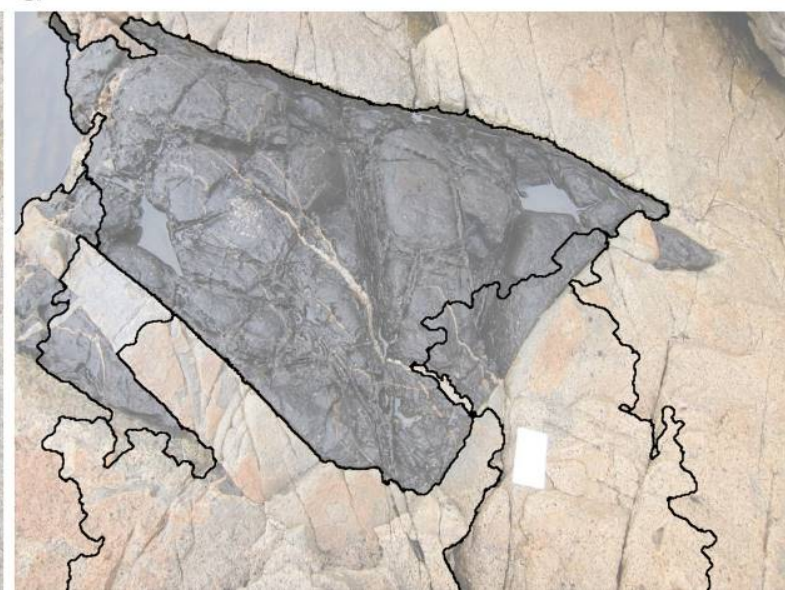

Figure 10. Lithological boundaries detected by DG using the user inputs shown in Figure 8.

\subsubsection{Experiments with non-geological images}

Images from the Berkeley database (Martin et al., 2001), Grabcut database (Rother et al., 2004), MSRM database (Ning et al., 2010) and the Microsoft Research database were used to analyse the performance of the proposed algorithm. The mean-shift algorithm (Comaniciu and Meer, 2002), implemented in the EDISON system was used as the initial segmentation algorithm for the proposed method and for MSRM. Since IGC, DG and MSRM do not have a boundary editing step, for fairness we did not use the boundary editing step of the ILBD method in these 
segmentations.

Figure 11 shows the segmentation results from the four algorithms. Visually these figures demonstrate the effectiveness of the proposed method in comparison to the others. To quantify the performance of algorithms the segmentation results were compared with ground truth images. For the first two images in Figure 11, the ground truth was not provided by the database thus we manually traced it and for the remaining four images the ground truth provided by the database is used. Table 2 shows the results of the quantitative analysis. 
Image
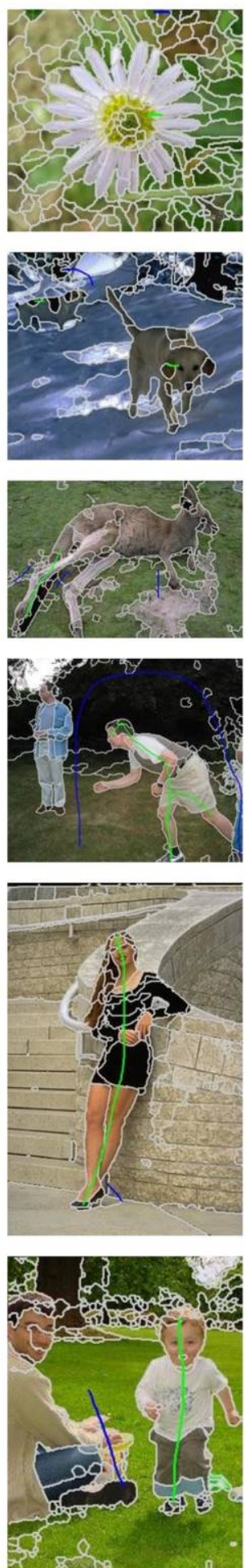

IGC

DG
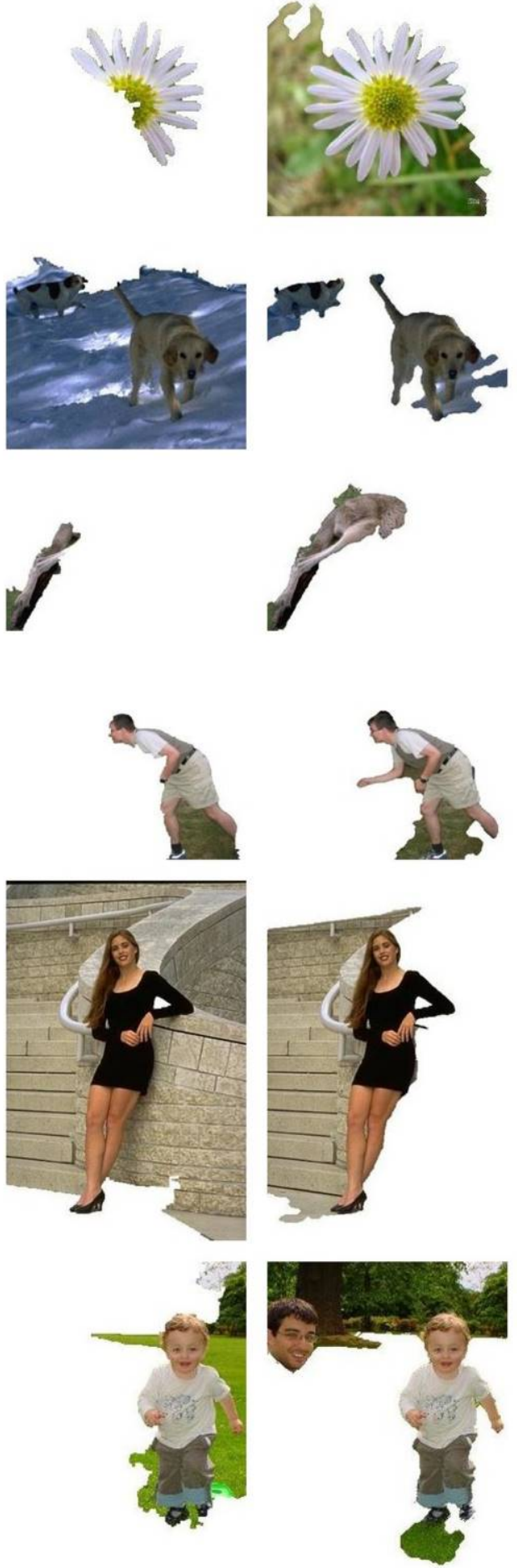

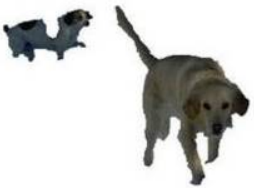

MSRM

ILBD
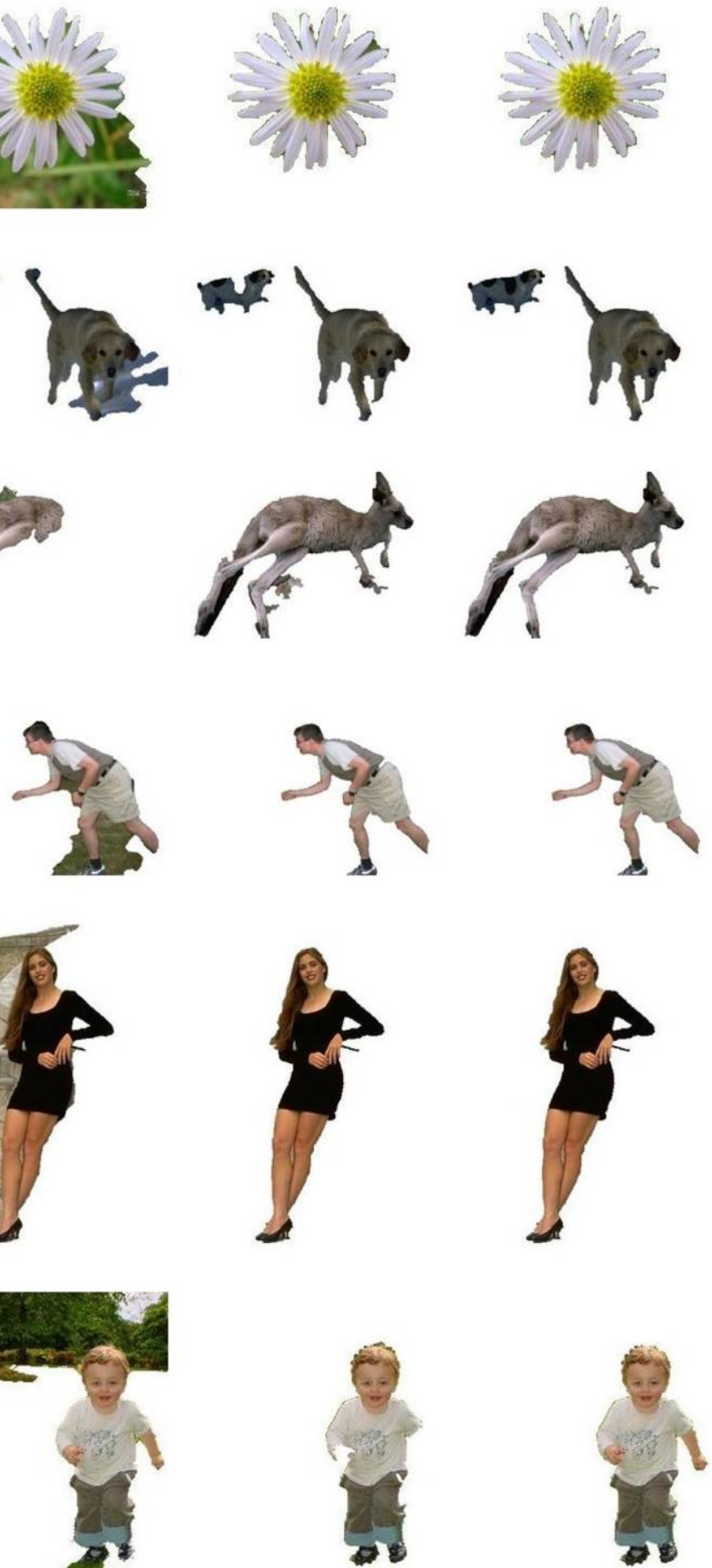

Figure 11. Comparison of segmentation results. First column: Initial segmentations and user input where the green line indicates the object of interest and blue indicates the background. Columns two to five: Object regions extracted by IGC, DG, MSRM and ILBD (without boundary editing step) respectively. 
Table 2. Quantitative results (Accuracy).

\begin{tabular}{cccccccc}
\hline Image & flower & twodogs & kangaroo & bool & woman & people & mean \\
\hline $\begin{array}{c}\text { size } \\
\text { (height*width) } \\
\text { Initial region }\end{array}$ & 216 X 229 & 295 X 335 & 321 X 481 & 450 X 520 & 481 X 321 & $320 \times 240$ & \\
& 250 & 196 & 204 & 223 & 252 & 318 & \\
MSRM & 98.54 & 98.87 & 97.23 & 99.25 & $\mathbf{9 9 . 2 6}$ & 98.48 & 98.60 \\
DG & 59.48 & 93.41 & 83.01 & 94.08 & 70.24 & 72.37 & 78.76 \\
IGC & 87.99 & 33.87 & 75.60 & 92.51 & 26.04 & 87.53 & 67.26 \\
ILBD & $\mathbf{9 8 . 7 1}$ & $\mathbf{9 9 . 1 7}$ & $\mathbf{9 8 . 1 1}$ & $\mathbf{9 9 . 3 1}$ & $\mathbf{9 9 . 2 6}$ & $\mathbf{9 9 . 5 7}$ & $\mathbf{9 9 . 0 2}$ \\
\hline
\end{tabular}

The proposed algorithm (ILBD) achieved a higher accuracy than all the other segmentation methods for all the images - achieving an average accuracy over 99\%. The MSRM performed better than the IGC and DG algorithms, but our ILBD method performed slightly better than the MSRM method. In the MSRM method, only the marked background regions are expanded firstly to extract the object from the image unlike the ILBD algorithm, where it expands all the marked regions with their adjacent regions if they have maximum similarity. Another main difference between the MSRM and ILBD methods is, in the region merging process, the MSRM algorithm forces all the non-marked regions to merge with one of their adjacent regions, but our ILBD method merges the adjacent regions only if their similarity is above the threshold. These differences may have contributed to the better performance of the ILBD method.

It is not possible to directly compare the time when analysing the performances of the algorithms, since the ILBD algorithm and MSRM are developed in MATLAB while DG is implemented in Java and IGC in $\mathrm{C}++$. The average time taken (without the time taken for user input) to produce the results (Figure 11) by the ILBD method is 8 seconds, while the MATLAB based MSRM algorithm took an average of 18 seconds.

\section{DISCUSSION}

\subsection{Limitations of proposed method}

The ILBD algorithm produces promising results for outcrop images captured at two different 
resolutions (Figure 9) but has the following limitations.

- The ILBD algorithm relies on the user's expertise in separating complex objects within images. A discussion on the potential biases and variability in mapping these lithological boundaries by a geologist is beyond the scope of this paper, nevertheless, the performance of the ILBD algorithm relies on the user's ability to sketch the marker lines over the areas, that capture the feature diversity in the lithological unit being mapped.

- Areas of shadow or regions that are occluded by vegetation or hidden under water will always be difficult to correctly classify. This is illustrated by the image in Figure 9c, where the algorithm failed to classify the boundary of granulite which is covered by grass. In Figure 9d, water is present but the user chose to label this as water rather than interpret the lithology beneath the water.

- In addition, our experiment showed that the results of the proposed ILBD algorithm depends heavily on the initial segments. If the initial segmentation groups some background and object pixels as the same region, the algorithm may fail to detect the boundary correctly, producing a misclassification that cannot be corrected by the boundary editing step. Thus the choice of the initial segmentation algorithm plays a critical role. In one of the experimental images, shown in figure 8c, the initial SLIC super pixels did not adhere to the lithological boundary well in some areas of the image. This is shown in the example area highlighted in Figure 12, where a portion of the clastbearing rhyolitic dike is grouped together with quartzo-feldspathic veins by the SLIC algorithm. Changing the parameters of this algorithm did not solve the problem. This may be addressed by using some other initial low level segmentation. 


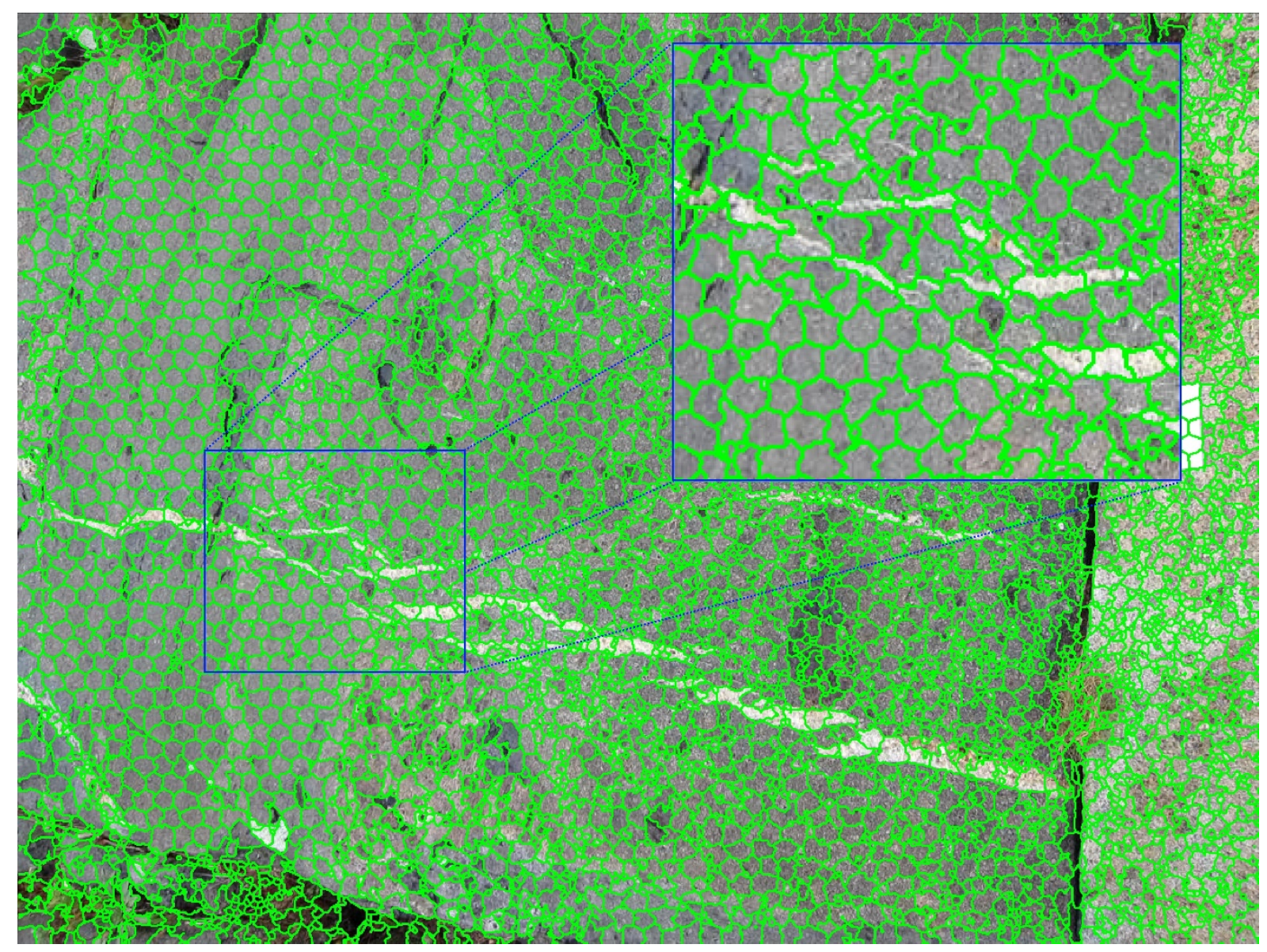

Figure 12. An example where the initial segments (SLIC superpixels) did not adhere to the boundary well.

\subsection{Future Development}

Further development of our ILBD method should involve: (1) Automated setting of parameters, especially for the colour similarity threshold for region merging. It is intended to apply adaptive thresholding for this purpose where the spatial variations in illumination are taken into account when deciding the threshold (Bradley and Roth, 2007). This thresholding method has been successfully applied to medical image segmentation (Saikumar et al., 2012; Stephanakis and Anastassopoulos, 2006). (2) Incorporation of texture along with colour for the region merging process, as texture is an important distinguishing characteristic for many rock types. This could be achieved by adapting the work of Chen et al., (2005), which uses colour and texture features for image segmentation. (3) Development of a platform, where photographic images can be integrated with other data types, such as radiometrics, thermal infrared, multi- or hyperspectral data. Previous studies showed that lithology units can be characterised and classified by machine learning algorithms applied to spectral images (Yu et al., 2012). (4) Applying this 
method to three dimensional (3D) point clouds to detect lithological boundaries in 3D. This could be achieved by adapting the work of previous studies which have successfully detected the geological structures (faults, joints and bedding) from 3D surface models (Lato and Vöge, 2012; Riquelme et al., 2014; Slob et al., 2005; Vasuki et al., 2014).

\section{CONCLUSION}

This paper presents an interactive image segmentation method specifically developed to map the lithological boundaries of complex geological images, the Interactive Lithological Boundary Detection method; ILBD. The proposed method uses an initial over segmented image in conjunction with user inputs to find detailed boundaries of multiple lithological units from images of exposed rock surfaces. The ILBD method also provides an important postprocessing boundary editing step to ensure the practical use of it by end-users. Our experimental results show that the ILBD method successfully separates lithologies in visually complex rock surface images. It generates outputs that are almost equivalent to that from manual mapping with more than $96 \%$ similarity, but in four times less time than that taken using standard digitising mapping methods. We also demonstrated that the proposed method outperforms three well known algorithms in segmenting natural images.

Our proposed lithology boundary mapping method, based on image analysis, offers an effective complementary approach to machine learning based lithology unit classification methods.

\section{ACKNOWLEDGMENT}

We acknowledge The University of Western Australia for providing a Scholarship for International Research Fees (SIRF) and an Ad Hoc Scholarship for this study. This work was also supported by the Australian Research Council linkage grant, LP140100267. We would like to acknowledge Darren Turner and Arko Lucieer, University of Tasmania, for the UAV images used in this study.

\section{REFERENCES}


Abdul-Qadir, A.M., 2013. Supervised Classification for Lithologic Discrimination in Shaikh Ibrahim Area, NW Iraq Using Landsat Images. Arab. J. Sci. Eng. 39, 437-451. doi:10.1007/s13369-013-0911-8

Achanta, R., Shaji, A., Smith, K., Lucchi, A., Fua, P., Susstrunk, S., 2012. SLIC superpixels compared to state-of-the-art superpixel methods. Pattern Anal. Mach. Intell. IEEE Trans. On 34, 2274-2282.

Bemis, S.P., Micklethwaite, S., Turner, D., James, M.R., Akciz, S., T. Thiele, S., Bangash, H.A., 2014. Ground-based and UAV-Based photogrammetry: A multi-scale, highresolution mapping tool for structural geology and paleoseismology. J. Struct. Geol. 69, 163-178. doi:10.1016/j.jsg.2014.10.007

Boykov, Y.Y., Jolly, M.-P., 2001. Interactive graph cuts for optimal boundary amp; region segmentation of objects in N-D images, in: Eighth IEEE International Conference on Computer Vision, 2001. ICCV 2001. Proceedings. Presented at the Eighth IEEE International Conference on Computer Vision, 2001. ICCV 2001. Proceedings, pp. 105-112 vol.1. doi:10.1109/ICCV.2001.937505

Bradley, D., Roth, G., 2007. Adaptive Thresholding using the Integral Image. J. Graph. GPU Game Tools 12, 13-21. doi:10.1080/2151237X.2007.10129236

Chen, J., Pappas, T.N., Mojsilović, A., Rogowitz, B.E., 2005. Adaptive perceptual colortexture image segmentation. IEEE Trans. Image Process. Publ. IEEE Signal Process. Soc. 14, 1524-1536.

Chen, M., Hou, Y., Zeng, X., Lu, Y., 2011. A multi-label interactive image segmentation method based on region merging, in: 2011 International Conference on Mechatronic Science, Electric Engineering and Computer (MEC). Presented at the 2011 International Conference on Mechatronic Science, Electric Engineering and Computer (MEC), pp. 2336-2340. doi:10.1109/MEC.2011.6025961

Cheng, Y., 1995. Mean shift, mode seeking, and clustering. IEEE Trans. Pattern Anal. Mach. Intell. 17, 790-799. doi:10.1109/34.400568

Comaniciu, D., Meer, P., 2002. Mean shift: a robust approach toward feature space analysis. IEEE Trans. Pattern Anal. Mach. Intell. 24, 603-619. doi:10.1109/34.1000236

Cracknell, M.J., Reading, A.M., 2013. The upside of uncertainty: Identification of lithology contact zones from airborne geophysics and satellite data using Random Forests and Support Vector Machines. Geophysics 78, WB113-WB126. doi:10.1190/geo20120411.1

Dhara, B.C., Chanda, B., 2011. A Fast Interactive Image Segmentation to Locate Multiple Similar-colored Objects, in: 2011 Third National Conference on Computer Vision, Pattern Recognition, Image Processing and Graphics (NCVPRIPG). Presented at the 2011 Third National Conference on Computer Vision, Pattern Recognition, Image Processing and Graphics (NCVPRIPG), pp. 25-28. doi:10.1109/NCVPRIPG.2011.13

EDISON system. (Accessed 2014 June 23) Retrieved from http://coewww.rutgers.edu/riul/research/code/EDISON/

Ferrero, A., Forlani, G., Roncella, R., Voyat, H., 2009. Advanced Geostructural Survey Methods Applied to Rock Mass Characterization. Rock Mech. Rock Eng. 42, 631665. doi:10.1007/s00603-008-0010-4

Ferrero, A.M., Migliazza, M., Roncella, R., Segalini, A., 2011. Rock cliffs hazard analysis based on remote geostructural surveys: The Campione del Garda case study (Lake Garda, Northern Italy). Geomorphology 125, 457-471. doi:10.1016/j.geomorph.2010.10.009

Gong, X., Liu, J., 2012. Rock detection via superpixel graph cuts, in: Image Processing (ICIP), 2012 19th IEEE International Conference on. IEEE, pp. 2149-2152. 
Grau, V., Mewes, A.U.J., Alcaniz, M., Kikinis, R., Warfield, S.K., 2004. Improved watershed transform for medical image segmentation using prior information. IEEE Trans. Med. Imaging 23, 447-458. doi:10.1109/TMI.2004.824224

Harwin, S., Lucieer, A., 2012. Assessing the Accuracy of Georeferenced Point Clouds Produced via Multi-View Stereopsis from Unmanned Aerial Vehicle (UAV) Imagery. Remote Sens. 4, 1573-1599. doi:10.3390/rs4061573

Jian, C., Bin, Y., Hua, J., Lei, Z., Li, T., 2013. Interactive image segmentation by improved maximal similarity based region merging, in: Medical Imaging Physics and Engineering (ICMIPE), 2013 IEEE International Conference on. IEEE, pp. 279-282.

Jones, G., 1994. Image segmentation using texture boundary detection. Pattern Recognit. Lett. 15, 533-541. doi:10.1016/0167-8655(94)90013-2

Jung, C., Jian, M., Liu, J., Jiao, L., Shen, Y., 2014. Interactive image segmentation via kernel propagation. Pattern Recognit. 47, 2745-2755. doi:10.1016/j.patcog.2014.02.010

Kottenstette, J., 2005. Measurement of Geologic Features using Close Range Terrestrial Photogrammetry, in: Alaska Rocks 2005, The 40th US Symposium on Rock Mechanics (USRMS).

Kovesi, P., 2015. Good Colour Maps: How to Design Them. ArXiv150903700 Cs.

Kovesi, P., 2013. MATLAB and Octave Functions for Computer Vision and Image Processing, http://www.peterkovesi.com/matlabfns/.

Lato, M.J., Vöge, M., 2012. Automated mapping of rock discontinuities in 3D lidar and photogrammetry models. Int. J. Rock Mech. Min. Sci. 54, 150-158. doi:10.1016/j.ijrmms.2012.06.003

Li, T., Xie, Z., Wu, J., Yan, J., Shen, L., 2013. Interactive object extraction by merging regions with k-global maximal similarity. Neurocomputing, Image Feature Detection and Description 120, 610-623. doi:10.1016/j.neucom.2013.04.009

Li, Y., Sun, J., Tang, C.-K., Shum, H.-Y., 2004. Lazy snapping, in: ACM Transactions on Graphics (ToG). ACM, pp. 303-308.

Liu, M.-Y., Tuzel, O., Ramalingam, S., Chellappa, R., 2011. Entropy rate superpixel segmentation, in: 2011 IEEE Conference on Computer Vision and Pattern Recognition (CVPR). Presented at the 2011 IEEE Conference on Computer Vision and Pattern Recognition (CVPR), pp. 2097-2104. doi:10.1109/CVPR.2011.5995323

Liu, X., Wang, D., 2006. Image and Texture Segmentation Using Local Spectral Histograms. IEEE Trans. Image Process. 15, 3066-3077. doi:10.1109/TIP.2006.877511

Long, J., Shen, X., Hui, Z., Chen, H., 2013. Superpixels Based Interactive Image Segmentation Algorithm, in: Proceedings of the 2013 International Conference on Information, Business and Education Technology (ICIBET 2013). Atlantis Press.

Lucieer, A., Robinson, S.A., Turner, D., 2011. Unmanned Aerial Vehicle (UAV) Remote Sensing for Hyperspatial Terrain Mapping of Antarctic Moss Beds based on Structure from Motion (SfM) point clouds. Proc. 34th Int. Symp. Remote Sens. Environ. ISRSE34.

Maiti, S., Tiwari, R.K., 2005. Automatic detection of lithologic boundaries using the Walsh transform: A case study from the KTB borehole. Comput. Geosci. 31, 949-955. doi:10.1016/j.cageo.2005.01.016

Martin, D., Fowlkes, C., Tal, D., Malik, J., 2001. A database of human segmented natural images and its application to evaluating segmentation algorithms and measuring ecological statistics, in: In Proc. 8th Int'l Conf. Computer Vision. pp. 416-423.

Martin, D.R., Fowlkes, C.C., Malik, J., 2004. Learning to detect natural image boundaries using local brightness, color, and texture cues. Pattern Anal. Mach. Intell. IEEE Trans. On 26, 530-549.

McGuinness, K., O’Connor, N.E., 2008. The K-Space segmentation tool set. 
Microsoft Research Databse. (Accessed 2014 July 20) Retrieved from http://research.microsoft.com/en-us/projects/ObjectClassRecognition

Mignotte, M., 2008. Segmentation by Fusion of Histogram-Based -Means Clusters in Different Color Spaces. IEEE Trans. Image Process. 17, 780-787. doi:10.1109/TIP.2008.920761

Moga, A.N., Gabbouj, M., 1998. Parallel Marker-Based Image Segmentation with Watershed Transformation. J. Parallel Distrib. Comput. 51, 27-45. doi:10.1006/jpdc.1998.1448

Ngcofe, L., Minnaar, H., 2012. A study on automated segmentation for object-based image analysis for geological mapping in the northern cape province, south africa., in: 4th GEOBIA. Rio de Janeiro - Brazil, p. p.129.

Ning, J., Zhang, L., Zhang, D., Wu, C., 2010. Interactive image segmentation by maximal similarity based region merging. Pattern Recognit. 43, 445-456. doi:10.1016/j.patcog.2009.03.004

Noma, A., Graciano, A.B.V., Cesar Jr, R.M., Consularo, L.A., Bloch, I., 2012. Interactive image segmentation by matching attributed relational graphs. Pattern Recognit. 45, 1159-1179. doi:10.1016/j.patcog.2011.08.017

Panagiotakis, C., Papadakis, H., Grinias, E., Komodakis, N., Fragopoulou, P., Tziritas, G., 2013. Interactive image segmentation based on synthetic graph coordinates. Pattern Recognit. 46, 2940-2952. doi:10.1016/j.patcog.2013.04.004

Peng, B., Zhang, L., Zhang, D., Yang, J., 2011. Image segmentation by iterated region merging with localized graph cuts. Pattern Recognit., Semi-Supervised Learning for Visual Content Analysis and Understanding 44, 2527-2538. doi:10.1016/j.patcog.2011.03.024

Perez, C.A., Saravia, J., Navarro, C., Castillo, L., Schulz, D., Aravena, C., 2012. Lithological classification based on Gabor texture image analysis, in: 2012 International Symposium on Optomechatronic Technologies (ISOT). Presented at the 2012 International Symposium on Optomechatronic Technologies (ISOT), pp. 1-3. doi:10.1109/ISOT.2012.6403273

Protiere, A., Sapiro, G., 2007. Interactive Image Segmentation via Adaptive Weighted Distances. IEEE Trans. Image Process. 16, 1046-1057. doi:10.1109/TIP.2007.891796

Riquelme, A.J., Abellán, A., Tomás, R., Jaboyedoff, M., 2014. A new approach for semiautomatic rock mass joints recognition from 3D point clouds. Comput. Geosci. 68, 38-52.

Rother, C., Kolmogorov, V., Blake, A., 2004. Grabcut: Interactive foreground extraction using iterated graph cuts, in: ACM Transactions on Graphics (TOG). ACM, pp. 309314.

Saikumar, T., Nagarani, M., Yojana, K., Shashidhar, B., 2012. Image segmentation of an adaptive threshold algorithm using watershed transform and fuzzy c-means clustering on level set method, in: 2012 International Conference on Advances in Engineering, Science and Management (ICAESM). Presented at the 2012 International Conference on Advances in Engineering, Science and Management (ICAESM), pp. 33-38.

Salati, S., van Ruitenbeek, F.J.A., van der Meer, F.D., Tangestani, M.H., van der Werff, H., 2011. Lithological mapping and fuzzy set theory: Automated extraction of lithological boundary from ASTER imagery by template matching and spatial accuracy assessment. Int. J. Appl. Earth Obs. Geoinformation 13, 753-765. doi:10.1016/j.jag.2011.05.004

Seers, T.D., Hodgetts, D., 2016. Probabilistic constraints on structural lineament best fit plane precision obtained through numerical analysis. J. Struct. Geol. 82, 37-47. doi:10.1016/j.jsg.2015.11.004 
Slob, S., van Knapen, B., Hack, R., Turner, K., Kemeny, J., 2005. Method for Automated Discontinuity Analysis of Rock Slopes with Three-Dimensional Laser Scanning. Transp. Res. Rec. J. Transp. Res. Board 1913, 187-194. doi:10.3141/1913-18

Song, Y.H., Shan, J., 2008. Automated rock segmentation for Mars Exploration Rover imagery, in: Lunar and Planetary Science Conference XXXIX, Houston, USA.

Stephanakis, I.M., Anastassopoulos, G.C., 2006. Segmentation using adaptive thresholding of the image histogram according to the incremental rates of the segment likelihood functions, in: Proceedings of 5th International Symposium Communication Systems Networks and Digital Signal Processing, University of Patras, Greece. pp. 464-468.

Taye, W., 2011. Lithological boundary detection using multi-sensor remote sensing imagery for geological interpretation (Master's thesis). University of Twente, The Netherlands.

Turner, D., Lucieer, A., Watson, C., 2012. An Automated Technique for Generating Georectified Mosaics from Ultra-High Resolution Unmanned Aerial Vehicle (UAV) Imagery, Based on Structure from Motion (SfM) Point Clouds. Remote Sens. 4, 1392-1410. doi:10.3390/rs4051392

van Ruitenbeek, F.J.A., van der Werff, H.M.A., Hein, K.A.A., van der Meer, F.D., 2008. Detection of pre-defined boundaries between hydrothermal alteration zones using rotation-variant template matching. Comput. Geosci. 34, 1815-1826. doi:10.1016/j.cageo.2007.11.001

Vantaram, S.R., Saber, E., 2012. Survey of contemporary trends in color image segmentation. J. Electron. Imaging 21, 040901-1.

Vasuki, Y., Holden, E.-J., Kovesi, P., Micklethwaite, S., 2014. Semi-automatic mapping of geological Structures using UAV-based photogrammetric data: An image analysis approach. Comput. Geosci. 69, 22-32. doi:10.1016/j.cageo.2014.04.012

Vezhnevets, V., Konouchine, V., 2004. "GrowCut” - Interactive Multi-Label ND Image Segmentation By Cellular Automata. ResearchGate 1.

Vincent, L., Soille, P., 1991. Watersheds in digital spaces: an efficient algorithm based on immersion simulations. IEEE Trans. Pattern Anal. Mach. Intell. 13, 583-598. doi:10.1109/34.87344

Vollgger, S.A., Cruden, A.R., 2016. Mapping folds and fractures in basement and cover rocks using UAV photogrammetry, Cape Liptrap and Cape Paterson, Victoria, Australia. J. Struct. Geol. 85, 168-187. doi:10.1016/j.jsg.2016.02.012

Yu, L., Porwal, A., Holden, E.-J., Dentith, M.C., 2012. Towards automatic lithological classification from remote sensing data using support vector machines. Comput. Geosci. 45, 229-239. doi:10.1016/j.cageo.2011.11.019

Zhou, C., Liu, C., 2012. Interactive Image Segmentation Based on Region Merging Using Hierarchical Match Mechanism, in: 2012 International Conference on Computer Science Service System (CSSS). Presented at the 2012 International Conference on Computer Science Service System (CSSS), pp. 1781-1784.

doi:10.1109/CSSS.2012.444 